Homology, Homotopy and Applications, vol. 8(2), 2006, pp.29-49

\title{
HOMOTOPY INVARIANCE IN E-THEORY
}

\author{
KLAUS THOMSEN
}

(communicated by G. Carlsson)

\begin{abstract}
We introduce equivalence relations among asymptotic homomorphisms that in general are stronger than homotopy, but which we show are equivalent to homotopy when the domain is a suspended $C^{*}$-algebra. As an application, we show that the E-theory of Connes and Higson can be realized as a special case of Kasparov's KK-theory.
\end{abstract}

\section{Introduction}

One of fundamental features in the Brown-Douglas-Fillmore theory of extensions is that the equivalence relation used to define the extension groups turns out to be homotopy invariant, see Theorem 2.14 of [3]. Similarly much of the power of Kasparov's generalization of the BDF-theory, cf. [12]-[14], comes from the fact that there are several equivalence relations on the fundamental objects, and only one of these relations is obviously homotopy invariant. The others are then shown to be homotopy invariant, and in fact to define the same relation, by means of the Kasparov product. This variety of apparently different equivalence relations is missing in the variant of KK-theory, called E-theory, which was introduced by Connes and Higson in [4]. The equivalence relation employed in the general Etheory framework has so far only been homotopy. But recently, the efforts towards classifying certain classes of $C^{*}$-algebras have met with the problem that while the objects of E-theory, i.e. the asymptotic homomorphisms, seem much more amenable to classification than the graded Hilbert $A-B$-modules of Kasparov, the equivalence relation - namely homotopy - is not. The most striking solution of this occurs in the classification of purely infinite simple nuclear $C^{*}$-algebras by Kirchberg and Phillips where a major part of the proof consists of realizing E-theory, for their particular class of $C^{*}$-algebras, as asymptotic homomorphisms modulo an equivalence relation which is (apparently) much stronger than homotopy, see [15], [23], [1]. Similar considerations and results can be found in the work of Lin, [17], [18] and Dadarlat and Eilers, [7].

The project of the present work is to transfer to asymptotic homomorphism the two most important equivalence relations which were used by Brown, Douglas, Fillmore and Kasparov and which are not obviously homotopy invariant. To describe what these relations become in E-theory, we formulate one of our main results:

Received October 17, 2003, revised October 10, 2005; published on April 21, 2006.

2000 Mathematics Subject Classification: 19K35, 46L85.

Key words and phrases: E-theory, asymptotic homomorphisms.

Copyright (C) 2006, International Press. Permission to copy for private use granted. 
Theorem 1.1. Let $A$ and $B$ be separable $C^{*}$-algebras, $B$ stable, and let

$$
\varphi=\left(\varphi_{t}\right)_{t \in[1, \infty)}, \quad \psi=\left(\psi_{t}\right)_{t \in[1, \infty)}: S A \rightarrow B
$$

be asymptotic homomorphisms. Then the following are equivalent:

(1) $[\varphi]=[\psi]$ in $[[S A, B]]$ (i.e. $\varphi$ and $\psi$ are homotopic).

(2) There is a family $\boldsymbol{\Phi}^{\lambda}: S A \rightarrow B, \lambda \in[0,1]$, of asymptotic homomorphisms such that $\boldsymbol{\Phi}^{0}=\varphi, \boldsymbol{\Phi}^{1}=\psi$, and the family of maps, $[0,1] \ni \lambda \mapsto \boldsymbol{\Phi}_{t}^{\lambda}(a), t \in[1, \infty)$, is equicontinuous for all $a \in S A$.

(3) There is an asymptotic homomorphism $\mu=\left(\mu_{t}\right)_{t \in[1, \infty)}: \operatorname{cone}(A) \rightarrow B$ and a norm-continuous path $U_{t}, t \in[1, \infty)$, of unitaries in $M_{2}(B)^{+}$such that

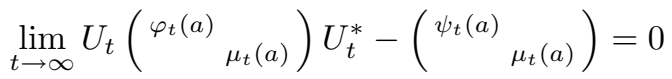

for all $a \in S A$.

Here, the equivalence relation described in (2) is the analog of operator homotopy while the equivalence relation described in (3) corresponds to unitary equivalence modulo addition by degenerate elements.

By Theorem 4.2 of [10], it is possible to realize KK-theory by using asymptotic homomorphisms where the individual maps are completely positive linear contractions. It is therefore interesting that we can improve condition (3) for such completely positive asymptotic homomorphisms in the following way: For given separable $C^{*}$-algebras $A$ and $B$, with $B$ stable, there is a completely positive asymptotic homomorphism $\lambda=\left(\lambda_{t}\right)_{t \in[1, \infty)}: \operatorname{cone}(A) \rightarrow B$ with the property that two completely positive asymptotic homomorphisms $\varphi=\left(\varphi_{t}\right)_{t \in[1, \infty)}, \psi=\left(\psi_{t}\right)_{t \in[1, \infty)}: S A$ $\rightarrow B$ are homotopic (as completely positive asymptotic homomorphisms) if and only if there is a norm-continuous path $U_{t}, t \in[1, \infty)$, of unitaries in $M_{2}(B)^{+}$and a continuous function $r:[1, \infty) \rightarrow[1, \infty)$ such that $\lim _{t \rightarrow \infty} r(t)=\infty$, and

$$
\lim _{t \rightarrow \infty} U_{t}\left(\begin{array}{ll}
\varphi_{t}(a) & \\
& \lambda_{r(t)}(a)
\end{array}\right) U_{t}^{*}-\left(\begin{array}{cc}
\psi_{t}(a) & \\
& \lambda_{r(t)}(a)
\end{array}\right)=0
$$

for all $a \in S A$.

As an application of the main results, we give in the final section a description of E-theory which shows, perhaps surprisingly, that E-theory is a specialization of KK-theory: For separable $C^{*}$-algebras $A$ and $B$, there is a natural isomorphism

$$
E(A, B) \simeq K K\left(A, C_{b}([1, \infty), B \otimes \mathbb{K}) / C_{0}([1, \infty), B \otimes \mathbb{K})\right) .
$$

Partial results in this direction were obtained by Nagy in [21]. Other applications of our main results can be found in $[\mathbf{2 7}]$ and $[\mathbf{2 8}]$.

In the first version of the present paper, a preprint entitled 'Homotopy invariance for bifunctors defined from asymptotic homomorphisms', the results were obtained for discrete asymptotic homomorphisms parallel with ordinary asymptotic homomorphisms. In the approach presented here, it is not necessary to consider discrete asymptotic homomorphisms, but the interested reader may consult [26] for a description of the relation between discrete and ordinary asymptotic homomorphisms. Another major improvement concerns the equivalence between (1) and (2) 
in Theorem 1.1 which in the preprint was proved by use of Higson's abstract version of Kasparovs homotopy invariance results, cf. [8]. Now a direct and quite simple proof exists and it was described in Theorem 3.4 of [20]. We can therefore concentrate here on the equivalence between (1) and (3). However, we end the paper with an example which shows that the implication $(1) \Rightarrow(2)$ of Theorem 1.1 can fail when the domain algebra is not a suspension.

\section{On absorbing extensions of a suspended $C^{*}$-algebra}

In this section, we present the key construction of the paper. The point of departure is the notion of absorbing $*$-homomorphisms, and we refer to [25] for the terminology and the basic results we shall need. We denote the multiplier algebra of a $C^{*}$-algebra $B$ by $\mathcal{M}(B)$.

Lemma 2.1. Let $A$ and $B$ be separable $C^{*}$-algebras with $B$ stable. Let $A_{0} \subseteq A$ be a hereditary $C^{*}$-subalgebra of $A$. If $\pi: A \rightarrow \mathcal{M}(B)$ is an absorbing *-homomorphism, then so is $\left.\pi\right|_{A_{0}}: A_{0} \rightarrow \mathcal{M}(B)$.

Proof. Let $\left\{u_{k}\right\}$ be an approximate unit in $A_{0}$, and define $R_{k}: A \rightarrow A_{0}$ by $R_{k}(a)=$ $u_{k} a u_{k}$. Then, the $R_{k}$ 's are completely positive contractions such that $\lim _{k \rightarrow \infty} R_{k}(x)$ $=x$ for all $x \in A_{0}$. By using this sequence, it follows easily that $\left(\left.\pi\right|_{A_{0}}\right)^{+}: A_{0}^{+} \rightarrow$ $\mathcal{M}(B)$ satisfies condition (1) of Theorem 2.1 in [25], cf. Lemma 2.1 of [28].

In this paper, we shall only use Lemma 2.1 in the case where the inclusion $A_{0} \subseteq A$ has the form $S A \subseteq \operatorname{cone}(A)$.

Given a Hilbert $B$-module $E$, we let $\mathbb{L}_{B}(E)$ denote the $C^{*}$-algebra of adjointable operators on $E$. The ideal of 'compact' operators in $\mathbb{L}_{B}(E)$ is denoted by $\mathbb{K}_{B}(E)$. In the special case where $E=B$, there are well-known identifications $\mathbb{L}_{B}(B)=\mathcal{M}(B)$ and $\mathbb{K}_{B}(B)=B$ which we shall use freely.

Assuming that $B$ is stable, we can choose a sequence $S_{i}, i=1,2, \ldots$, of isometries in $\mathcal{M}(B)$ with orthogonal ranges such that $\sum_{i=1}^{\infty} S_{i} S_{i}^{*}=1$, where the sum converges in the strict topology. If $\pi: A \rightarrow \mathcal{M}(B)$ is a $*$-homomorphism, we can then form a new $*$-homomorphism $\pi^{\infty} \oplus 0^{\infty}: A \rightarrow \mathcal{M}(B)$ which is given by $\left(\pi^{\infty} \oplus 0^{\infty}\right)(a)=$ $\sum_{i=1}^{\infty} S_{2 i} \pi(a) S_{2 i}^{*}$.

Definition 2.2. A $*$-homomorphism $\pi: A \rightarrow \mathcal{M}(B)$ is saturated when $\pi$ is unitarily equivalent to $\pi^{\infty} \oplus 0^{\infty}$.

Lemma 2.3. Let $A$ and $B$ be separable $C^{*}$-algebras with $B$ stable. Let $\pi: A \rightarrow$ $\mathcal{M}(B)$ be a saturated and absorbing $*$-homomorphism. Let $X$ be a compact metrizable space with base-point $x_{0} \in X$ and set $C_{0}(X)=\left\{f \in C(X): f\left(x_{0}\right)=0\right\}$. Define $1_{C_{0}(X)} \otimes \pi: A \rightarrow \mathcal{M}\left(C_{0}(X) \otimes B\right) \quad$ by $\quad\left(1_{C_{0}(X)} \otimes \pi(a) f\right)(x)=\pi(a) f(x), \quad x \in X$, $f \in C_{0}(X) \otimes B$. Then $1_{C_{0}(X)} \otimes \pi$ is absorbing.

Proof. By Theorem 2.1 of [25], it suffices to consider a completely positive contraction $\varphi: A^{+} \rightarrow C_{0}(X) \otimes B$, finite subsets $F \subseteq A^{+}, G \subseteq C_{0}(X) \otimes B$ and $\epsilon>0$, and 
construct $L \in \mathcal{M}\left(C_{0}(X) \otimes B\right)$ such that $\left\|L^{*} g\right\|<\epsilon, g \in G$, and

$$
\left\|\varphi(a)-L^{*}\left(1_{C_{0}(X)} \otimes \pi\right)^{+}(a) L\right\|<\epsilon
$$

for all $a \in F$. There is a finite set $x_{1}, x_{2}, x_{3}, \ldots, x_{n}$ in $X \backslash\left\{x_{0}\right\}$ and a partition of unity $\left\{h_{i}: i=1,2, \ldots, n\right\}$ in $C(X)$ such that $\left\|\varphi(a)-\sum_{i=1}^{n} h_{i} \varphi(a)\left(x_{i}\right)\right\|<\frac{\epsilon}{2}, a \in F$. Since $\pi$ is saturated, there is a sequence of isometries $T_{i}, i \in \mathbb{N}$, in $\mathcal{M}(B)$ such that $T_{i}^{*} \pi^{+}\left(A^{+}\right) T_{j}=\{0\}, \quad i \neq j, T_{i}^{*} \pi^{+}(a) T_{i}=\pi^{+}(a)$ for all $i, a$, and $\lim _{k \rightarrow \infty}\left\|T_{k}^{*} b\right\|=$ 0 for all $b \in B$. Since $\{g(x): x \in X, g \in G\}$ is a compact subset of $B$ and $\pi^{+}$ is unitally absorbing, it follows from Theorem 2.1 of [25] that we can find elements $V_{1}, V_{2}, \ldots, V_{n} \in \mathcal{M}(B)$ such that $\left\|V_{i}^{*} \pi^{+}(a) V_{i}-\varphi(a)\left(x_{i}\right)\right\|<\frac{\epsilon}{2}, \quad a \in F$, $i=1,2, \ldots, n$. Set $W_{i}=T_{K+i} V_{i}, i=1,2, \ldots, n$. If $K$ is large enough, we have that $\left\|W_{i}^{*} \pi^{+}(a) W_{i}-\varphi(a)\left(x_{i}\right)\right\|<\frac{\epsilon}{2}, \quad a \in F, W_{i}^{*} \pi^{+}\left(A^{+}\right) W_{j}=\{0\}, \quad i \neq j$, and $\left\|W_{i}^{*} g(x)\right\|<\frac{\epsilon}{n}, g \in G, x \in X$. Define the desired $L$ by

$$
(L f)(x)=\sum_{i=1}^{n} \sqrt{h_{i}(x)} W_{i} f(x) .
$$

In the following, we will let $1_{m}$ and $0_{m}$ denote the unit and the zero element of $M_{m}(\mathcal{M}(B))$, respectively. We will identify $M_{m}(\mathcal{M}(B))$ and $\mathcal{M}\left(M_{m}(B)\right)$.

Lemma 2.4. Let $D$ and $B$ be $C^{*}$-algebras, $B$ separable. Let $\pi: D \rightarrow \mathcal{M}(B)$ be a *-homomorphism and $p \in \mathcal{M}(B)$ a projection such that $p \pi(D) \subseteq B$. Assume that $F \subseteq D$ is a finite set and $\delta>0$ is such that

$$
\|\pi(a) p-p \pi(a)\|<\delta, a \in F .
$$

Let $F_{1} \subseteq D$ and $G \subseteq B$ be finite sets. Let $0 \leqslant z \leqslant 1$ be a strictly positive element in $(1-p) B(1-p)$ and let $\left.\epsilon_{1}, \epsilon_{2} \in\right] 0,1[$ be given. There is then a continuous function $g:[0,1] \rightarrow[0,1]$ such that $g$ is zero in a neighborhood of $0, g(t)=1, t \geqslant \epsilon_{1}$,

$$
\begin{gathered}
\sup _{t \in[0,1]}\|[\pi(d), p+g(t z)]\|<5 \delta, \quad d \in F, \\
\|[\pi(d), p+g(z)]\|<\epsilon_{2}, \quad d \in F_{1},
\end{gathered}
$$

and

$$
\|p b+g(z) b-b\|<\epsilon_{2}, \quad b \in G .
$$

Proof. Let $\Lambda$ denote the convex set of continuous functions $g:[0,1] \rightarrow[0,1]$ such that $g$ is zero in a neighborhood of 0 and $g(t)=1, t \geqslant \epsilon_{1}$. For each $x \in F$, define a multiplier $\tilde{x}$ of cone $((1-p) B(1-p))$ by $(\tilde{x} f)(t)=(1-p) \pi(x)(1-p) f(t), t \in[0,1]$, and define $\tilde{g} \in \operatorname{cone}((1-p) B(1-p))$ by $\tilde{g}(t)=g(t z)$. Since $t \mapsto t z$ is a strictly positive element of cone $((1-p) B(1-p)),\{(\tilde{g}, g(z)): g \in \Lambda\}$, is a convex approximate unit in cone $((1-p) B(1-p)) \oplus(1-p) B(1-p)$. Since $\pi(D) p \subseteq B$, we can use the argument from the proof of the existence of quasi-central approximate units, cf. [2], 
to find a $g \in \Lambda$ such that

$$
\|[(\tilde{x}, \pi(y)),(\tilde{g}, p+g(z))]\|<\min \left\{\delta, \epsilon_{2}\right\},
$$

for all $x \in F, y \in F_{1}$, and

$$
\|p b+g(z) b-b\|<\epsilon_{2}, b \in G .
$$

For completeness, we include the argument: First observe that

$$
\begin{aligned}
\|p b+g(z) b-b\|^{2} & =\|g(z)(1-p) b-(1-p) b\|^{2}=\|(g(z)-1)(1-p) b\|^{2} \\
& =\left\|(g(z)-1)(1-p) b b^{*}(1-p)(g(z)-1)\right\|
\end{aligned}
$$

tends to 0 as $g \rightarrow 1$, increasingly, for all $b \in B$. In particular, there is a $g_{0} \in \Lambda$ such that $\|p b+g(z) b-b\|<\epsilon_{2}, b \in G$, for all $g \geqslant g_{0}$. Set $E=\operatorname{cone}((1-p) B(1-p)) \oplus$ $B, m_{g}=(\tilde{g}, p+g(z))$, and consider a pair $x \in F, y \in F_{1}$. Note that

$$
\left[(\tilde{x}, \pi(y)), m_{g}\right] \in E
$$

for all $g \in \Lambda$ since $\pi(D) p \subseteq B$. Let $\varphi$ be any state on $E$ and $\left(\pi_{\varphi}, H_{\varphi}, \xi_{\varphi}\right)$ the corresponding cyclic representation of $E$, cf. Theorem 3.3.3 of [22]. Being cyclic, $\pi_{\varphi}$ is also non-degenerate and extends therefore to a representation $\overline{\pi_{\varphi}}$ of $\mathcal{M}(E)$. Note that $\lim _{g \in \Lambda} \overline{\pi_{\varphi}}\left(m_{g}\right)=1$ in the strong operator topology because $\lim _{g \in \Lambda} m_{g} e=e$ for all $e \in E$. It follows that

$$
\begin{aligned}
\lim _{g \in \Lambda} \varphi\left(\left[(\tilde{x}, \pi(y)), m_{g}\right]\right)= & \lim _{g \in \Lambda}\left\langle\pi_{\varphi}\left(\left[(\tilde{x}, \pi(y)), m_{g}\right]\right) \xi_{\varphi}, \xi_{\varphi}\right\rangle \\
= & \lim _{g \in \Lambda}\left(\left\langle\overline{\pi_{\varphi}}((\tilde{x}, \pi(y))) \overline{\pi_{\varphi}}\left(m_{g}\right) \xi_{\varphi}, \xi_{\varphi}\right\rangle\right. \\
& \left.-\left\langle\overline{\pi_{\varphi}}((\tilde{x}, \pi(y))) \xi_{\varphi}, \overline{\pi_{\varphi}}\left(m_{g}\right) \xi_{\varphi}\right\rangle\right) \\
= & 0 .
\end{aligned}
$$

Since $\varphi$ was arbitrary, it follows that 0 is in the weak closure of $\left\{\left[(\tilde{x}, \pi(y)), m_{g}\right]\right.$ : $\left.g \in \Lambda, g \geqslant g_{0}\right\}$ in $E$. But the latter set is convex; so also the norm-closure of it contains 0 , i.e. there is a $g \geqslant g_{0}$ such that (5) holds for the pair $(x, y) \in F \times F_{1}$. The same argument applied to $E^{\# F \# F_{1}}$ instead of $E$ shows that we can make (5) hold for all $(x, y) \in F \times F_{1}$ and some $g \geqslant g_{0}$ in $\Lambda$.

(4) holds because $g \geqslant g_{0}$ and (3) follows from (5) which also implies that

$$
\sup _{t \in[0,1]}\|[(1-p) \pi(x)(1-p), g(t z)]\|<\delta, x \in F \text {. }
$$

Since

$$
\begin{aligned}
& {[\pi(x), g(t z)]=} \\
& \quad[(1-p) \pi(x)(1-p), g(t z)]+[(1-p) \pi(x) p, g(t z)]+[p \pi(x)(1-p), g(t z)],
\end{aligned}
$$

we get (2) by combining (7) with (1).

Let $\mathcal{H}$ be an infinite-dimensional separable Hilbert space. We can then define $g:[0, \infty[\rightarrow[0,2]$ by

$$
g(s)=\sup \{\|[a, \sqrt{x}]\|: a, x \in \mathcal{B}(\mathcal{H}),\|a\| \leqslant 1,0 \leqslant x \leqslant 1,\|[a, x]\| \leqslant s\} .
$$

By the Lemma on page 332 of [2], $g$ is continuous at 0, i.e. $\lim _{s \rightarrow 0} g(s)=0 . g$ will feature in the next Lemma. 
Lemma 2.5. Let $D$ and $B$ be separable $C^{*}$-algebras with $D$ contractible. Let $\varphi_{t}$ : $D \rightarrow D, t \in[0,1]$, be a homotopy of endomorphisms of $D$ such that $\varphi_{0}=\mathrm{id}$ and $\varphi_{1}=0$. Let $F_{0} \subseteq F_{1} \subseteq D$ and $G_{1} \subseteq B$ be finite subsets. Let $\pi: D \rightarrow \mathcal{M}(B)$ be a*homomorphism and $p \in \mathcal{M}(B)$ a projection such that $p \pi(D) \subseteq B$ and $\| p \pi\left(\varphi_{t}(a)\right)-$ $\pi\left(\varphi_{t}(a)\right) p \|<\kappa, a \in F_{0}, t \in[0,1]$, for some $\kappa>0$.

For any $\epsilon>0$, there is then an $n \in \mathbb{N}$, a*-homomorphism $\pi_{1}: D \rightarrow \mathcal{M}\left(M_{n}(B)\right)$ and a continuous path $p_{t}, t \in[0,1]$, of elements $p_{t} \in \mathcal{M}\left(M_{n+1}(B)\right)$ such that

(1) $0 \leqslant p_{t} \leqslant 1, t \in[0,1]$,

(2) $\left(p_{t}^{2}-p_{t}\right)\left(\begin{array}{ll}\pi(a) & \\ & \pi_{1}(a)\end{array}\right)=0, \quad a \in D, t \in[0,1]$

(3) $p_{t}\left(\begin{array}{ll}\pi(a) \\ & \pi_{1}(a)\end{array}\right) \in M_{n+1}(B), a \in D, t \in[0,1]$,

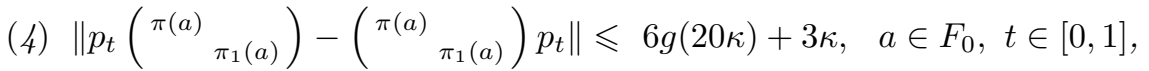

(5) $\left(\begin{array}{ll}p & \\ & 0_{n}\end{array}\right) \leqslant p_{t}, t \in[0,1]$,

(6) $\left\|p_{1}\left(\begin{array}{ll}\pi\left(\varphi_{t}(a)\right) & \\ & \pi_{1}\left(\varphi_{t}(a)\right)\end{array}\right)-\left(\begin{array}{ll}\pi\left(\varphi_{t}(a)\right) & \\ & \pi_{1}\left(\varphi_{t}(a)\right)\end{array}\right) p_{1}\right\| \leqslant \epsilon, \quad a \in F_{1}, \quad t \in[0,1]$,

(7) $\left\|p_{1}\left(\begin{array}{cc}b & \\ { }^{n}\end{array}\right)-\left(\begin{array}{cc}b & \\ { }^{n} & 0_{n}\end{array}\right)\right\| \leqslant \epsilon, b \in G_{1}$,

(8) $p_{1}=p_{1}^{2}, p_{0}=\left(\begin{array}{cc}p & \\ & 0_{n}\end{array}\right)$.

Proof. The proof is an elaboration of Voiculescus' proof of Proposition 3 in [29]. Let $\delta>0$ be so small that $6 g(4 \delta)+3 \delta<\frac{\epsilon}{3}, \delta<\kappa$ and $\delta+\sqrt{\|b\| \delta}<\epsilon$ for all $b \in G_{1}$. Choose first a finite $\frac{\epsilon}{3}$-dense subset $F$ of $\left\{\varphi_{t}(a): t \in[0,1], a \in F_{1}\right\}$, and then a $n$ so large that $t, s \in[0,1],|s-t| \leqslant 1 / n \Rightarrow\left\|\varphi_{t}(a)-\varphi_{s}(a)\right\|<\delta, a \in F$. Let $0 \leqslant z \leqslant 1$ be a strictly positive element in $(1-p) B(1-p)$. It follows from Lemma 2.4 that there are continuous functions $g_{i}:[0,1] \rightarrow[0,1], i=0,1, \ldots, n-1$, which are all zero in a neighborhood of 0 such that $g_{j} g_{j-1}=g_{j-1}, j=1,2, \ldots, n-1$, and such that the elements $x_{j}=p+g_{j}(z)$ and $x_{j}^{t}=p+g_{j}(t z)$ satisfy that

$$
\left\|x_{j} \pi \circ \varphi_{\frac{j}{n}}(a)-\pi \circ \varphi_{\frac{j}{n}}(a) x_{j}\right\| \leqslant \delta
$$

$j=0,1,2, \cdots, n-1, a \in F,\left\|x_{0} b-b\right\| \leqslant \delta, b \in G_{1}$, and

$$
\left\|x_{j}^{t} \pi \circ \varphi_{\frac{j}{n}}(a)-\pi \circ \varphi_{\frac{j}{n}}(a) x_{j}^{t}\right\|<5 \kappa
$$

$j=0,1,2, \cdots, n-1, a \in F_{0}, t \in[0,1]$. Set $\pi_{1}=\operatorname{diag}\left(\pi \circ \varphi_{\frac{1}{n}}, \pi \circ \varphi_{\frac{2}{n}}, \cdots, \pi \circ \varphi_{1}\right)$ and

$$
p_{t}=\left(\begin{array}{ccc}
p & & \\
& 0 & \\
& & 2 t\left(1_{1}-p\right)
\end{array}\right), \quad t \in\left[0, \frac{1}{2}\right] .
$$

Then (1)-(5) hold trivially for $t \in\left[0, \frac{1}{2}\right]$. Note that $x_{i}^{t} x_{i-1}^{t}=x_{i-1}^{t}, i=1, \ldots, n-1$. Set $X_{t}^{0}=x_{0}^{2 t-1}, X_{t}^{j}=x_{j}^{2 t-1}-x_{j-1}^{2 t-1}, j=1,2, \ldots, n-1$, and $X_{t}^{n}=1_{1}-x_{n-1}^{2 t-1}$, 
$t \in\left[\frac{1}{2}, 1\right]$. Define $T_{t} \in \mathcal{M}\left(M_{n+1}(B)\right), t \in\left[\frac{1}{2}, 1\right]$, by

$$
T_{t}=\left(\begin{array}{cccc}
\sqrt{X_{t}^{0}} & 0 & \ldots & 0 \\
\sqrt{X_{t}^{1}} & 0 & \ldots & 0 \\
\vdots & \vdots & \ddots & \vdots \\
\sqrt{X_{t}^{n}} & 0 & \ldots & 0
\end{array}\right) .
$$

Then $T_{t} T_{t}^{*}$ is a projection since $T_{t}^{*} T_{t}$ clearly is. Since $T_{\frac{1}{2}} T_{\frac{1}{2}}^{*}=p_{\frac{1}{2}}$ we can extend $p_{t}, t \in\left[0, \frac{1}{2}\right]$, to a continuous path in $\mathcal{M}\left(M_{n+1}(B)\right)$ by setting $p_{t}=T_{t} T_{t}^{*}, t \in\left[\frac{1}{2}, 1\right]$. Then (1) and (2) clearly hold and (3) follows from the observation that

$$
\left(\begin{array}{ll}
\pi(a) & \\
& \pi_{1}(a)
\end{array}\right) T_{t} \subseteq M_{n+1}(B), a \in D, t \in\left[\frac{1}{2}, 1\right] .
$$

It follows from (8) and (9), by using that $T_{t} T_{t}^{*}$ is tri-diagonal as in the proof of Proposition 3 in [29], that

$$
\left\|\left[p_{1},\left(\begin{array}{ll}
\pi(a) & \\
& \pi_{1}(a)
\end{array}\right)\right]\right\| \leqslant 6 g(4 \delta)+3 \delta \leqslant \frac{\epsilon}{3}, a \in F,
$$

and

$$
\left\|\left[p_{t},\left(\begin{array}{cc}
\pi(a) & \\
& \pi_{1}(a)
\end{array}\right)\right]\right\| \leqslant 6 g(20 \kappa)+3 \kappa, a \in F_{0}, t \in\left[\frac{1}{2}, 1\right],
$$

i.e. (4) and (6) hold. (5) is trivial when $t \in\left[0, \frac{1}{2}\right]$ and for $t>\frac{1}{2}$, it follows from the observation that

$$
\left(\begin{array}{cc}
p & \\
& 0_{n}
\end{array}\right) T_{t}=\left(\begin{array}{cc}
p & \\
& 0_{n}
\end{array}\right), \quad\left(\begin{array}{cc}
p & \\
& 0_{n}
\end{array}\right) T_{t}^{*}=\left(\begin{array}{cc}
p & \\
& 0_{n}
\end{array}\right) .
$$

It is straightforward to check that $\left\|p_{1}\left(\begin{array}{cc}b & { }_{0}\end{array}\right)-\left(\begin{array}{cc}{ }^{b}{ }_{0_{n}}\end{array}\right)\right\| \leqslant\left\|X_{1}^{0} b-b+\sqrt{X_{1}^{1}} \sqrt{X_{1}^{0}} b\right\|$ $\leqslant \delta+\sqrt{\|b\| \delta}$ when $b \in G_{1}$, and (7) holds. (8) is trivial.

Theorem 2.6. Let $A$ and $B$ be separable $C^{*}$-algebras, $B$ stable. There exists a saturated and absorbing $*$-homomorphism $\pi: \operatorname{cone}(A) \rightarrow \mathcal{M}(B)$ such that also $\left.\pi\right|_{S A}$ : $S A \rightarrow \mathcal{M}(B)$ is saturated and absorbing, and a continuous path $p_{t}, t \in[0, \infty)$, of elements in $\mathcal{M}(B)$ such that

(1) $0 \leqslant p_{t} \leqslant 1, \quad t \in[0, \infty)$,

(2) $p_{t} \pi(\operatorname{cone}(A)) \subseteq B, \quad t \in[0, \infty)$,

(3) $\left(p_{t}^{2}-p_{t}\right) \pi(\operatorname{cone}(A))=\{0\}, \quad t \in[0, \infty)$,

(4) $\lim _{t \rightarrow \infty} p_{t} b=b, \quad b \in B$,

(5) $\lim _{t \rightarrow \infty}\left\|p_{t} \pi(a)-\pi(a) p_{t}\right\|=0, \quad a \in \operatorname{cone}(A)$,

(6) $p_{0}=0, p_{n}^{2}=p_{n}, n=1,2,3, \ldots$

Proof. By [25] and Lemma 2.1, there is an absorbing *-homomorphism $S A \rightarrow$ $\mathcal{M}(B)$ which is the restriction of an absorbing $*$-homomorphism $\Theta: \operatorname{cone}(A) \rightarrow$ $\mathcal{M}(B)$. Let $F_{1} \subseteq F_{2} \subseteq F_{3} \subseteq \cdots$ and $G_{1} \subseteq G_{2} \subseteq G_{3} \subseteq \cdots$ be sequences of finite sets with dense union in $\operatorname{cone}(A)$ and $B$, respectively. By using Lemma 2.5, we can construct a sequence $1=n_{0}<n_{1}<n_{2}<\cdots$ of natural numbers, paths $p_{i}(t)$, $t \in[i-1, i]$, in $M_{n_{i}}(\mathcal{M}(B)), i=1,2, \ldots$, and $*$-homomorphisms $\tilde{\pi}_{i}: \operatorname{cone}(A) \rightarrow$ 
$M_{n_{i}-n_{i-1}}(\mathcal{M}(B)), i=1,2, \ldots$, such that $\pi_{0}=\Theta$ and $\pi_{i}=\left(\begin{array}{ll}\pi_{i-1} & \\ & \tilde{\pi}_{i}\end{array}\right): \operatorname{cone}(A) \rightarrow$ $M_{n_{i}}(\mathcal{M}(B)), i=1,2, \ldots$, satisfy

(1) $0 \leqslant p_{i}(t) \leqslant 1, t \in[i-1, i], i=1,2, \ldots$,

(2) $\left(p_{i}(t)^{2}-p_{i}(t)\right) \pi_{i}(\operatorname{cone}(A))=\{0\}, t \in[i-1, i], i=1,2, \ldots$,

(3) $p_{i}(t) \pi_{i}(\operatorname{cone}(A)) \subseteq M_{n_{i}}(B), t \in[i-1, i], i=1,2, \ldots$,

(4) $p_{i}(t) \geqslant\left(\begin{array}{cc}p_{i-1}(i-1) & 0 \\ 0 & 0_{n_{i}-n_{i-1}}\end{array}\right), t \in[i-1, i], i=2,3, \ldots$,

(5) $\left\|p_{i}(i)\left(\begin{array}{cc}b & \\ { }^{n} n_{n_{i}-n_{i-1}}\end{array}\right)-\left(\begin{array}{cc}b & \\ { }^{0} 0_{n_{i}-n_{i-1}}\end{array}\right)\right\| \leqslant \frac{1}{i}$, when all entries of $b \in M_{n_{i-1}}(B)$ come from $G_{i}, i=1,2,3, \ldots$,

(6) $\left\|p_{i}(t) \pi_{i}(a)-\pi_{i}(a) p_{i}(t)\right\| \leqslant \frac{1}{i}, a \in F_{i}, t \in[i-1, i], i=1,2, \ldots$,

(7) $p_{i}(i-1)=p_{i}(i-1)^{2}=\left(\begin{array}{cc}p_{i-1}(i-1) & 0 \\ 0 & 0_{n_{i}-n_{i-1}}\end{array}\right), i=2,3, \ldots$,

and $p_{1}(0)=0$. The construction can proceed by induction if the following condition is added-in the notation from Lemma 2.5:

$$
\text { i) } \quad\left\|p_{i}(i) \pi_{i}\left(\varphi_{t}(a)\right)-\pi_{i}\left(\varphi_{t}(a)\right) p_{i}(i)\right\| \leqslant \delta_{i+1},
$$

for $a \in F_{i+1}, t \in[0,1]$, where $\delta_{i+1}>0$ is chosen such that $6 g\left(20 \delta_{i+1}\right)+3 \delta_{i+1} \leqslant \frac{1}{i+1}$. Note that we can arrange that $\tilde{\pi}_{i}$ has the form $\tilde{\pi}_{i}=\pi_{i-1} \oplus \varphi_{i} \oplus 0_{n_{i-1}}$ for some *-homomorphism $\varphi_{i}: \operatorname{cone}(A) \rightarrow M_{n_{i}-2 n_{i-1}}(\mathcal{M}(B))$. Now define $\varphi^{\prime}: \operatorname{cone}(A) \rightarrow$ $\mathbb{L}_{B}\left(l_{2}(B)\right)$ by

$$
\varphi^{\prime}(d)=\operatorname{diag}\left(\Theta(d), \widetilde{\pi_{1}}(d), \widetilde{\pi_{2}}(d), \widetilde{\pi_{3}}(d), \ldots\right)
$$

and set

$$
p_{t}^{\prime}=\left(\begin{array}{cc}
p_{i}(t) & \\
& 0_{\infty}
\end{array}\right), t \in[i-1, i], i=1,2, \ldots .
$$

$\varphi^{\prime}$ is unitarily equivalent to a $*$-homomorphism $\pi: \operatorname{cone}(A) \rightarrow \mathcal{M}(B)$ since $l_{2}(B) \simeq$ $B$ as Hilbert $B$-modules. Note that both $\pi$ and $\left.\pi\right|_{S A}: S A \rightarrow \mathcal{M}(B)$ are absorbing because $\Theta$ has these properties. Furthermore, both $\pi$ and $\left.\pi\right|_{S A}$ are saturated since each $\pi_{i}$ as well as 0 occur as direct summands in $\widetilde{\pi_{k}}$ for infinitely many $k$ 's. Via the isomorphism $l_{2}(B) \simeq B, p^{\prime}$ becomes a path $p_{t}, t \in[0, \infty)$, in $\mathcal{M}(B)$ which has the properties (1)-(6) stated in the theorem.

\section{Duality in KK-theory}

Let $A$ and $B$ be separable $C^{*}$-algebras, with $B$ stable. Consider an absorbing *-homomorphism $\pi: A \rightarrow \mathcal{M}(B)$ and set

$$
\begin{gathered}
\mathcal{A}=\{x \in \mathcal{M}(B): x \pi(a)-\pi(a) x \in B, a \in A\}, \\
\mathcal{B}=\{x \in \mathcal{M}(B): x \pi(a), \pi(a) x \in B, a \in A\} .
\end{gathered}
$$

Then $\mathcal{B}$ is an ideal in $\mathcal{A}$, and in [25], it was shown that

$$
K_{1}(\mathcal{A} / \mathcal{B})=K K(A, B) .
$$


This identification is given by associating to a unitary $u \in M_{n}(\mathcal{A} / \mathcal{B})$ the Kasparov $A, B$-bimodule

$$
\left(B^{n} \oplus B^{n},\left(\pi^{n} \pi^{n}\right),\left(v^{*} v\right)\right),
$$

where $\pi^{n}(a)=\operatorname{diag}(\pi(a), \pi(a), \cdots, \pi(a)), B^{n} \oplus B^{n}$ is graded by $(x, y) \rightarrow(x,-y)$ and $v \in M_{n}(\mathcal{A})$ is any lift of $u$. In order to use the constructions from the last section, it is helpful to improve a little on this correspondence between $\mathrm{KK}$ and $K_{1}$, as follows.

Lemma 3.1. $K_{0}(\mathcal{B})=K_{1}(\mathcal{B})=0$.

Proof. Consider

$$
\mathcal{B}_{1}=\left\{X \in M_{2}(\mathcal{M}(B)): X\left(\begin{array}{cc}
\pi(a) & \\
& 0
\end{array}\right),\left(\begin{array}{ll}
\pi(a) & \\
& 0
\end{array}\right) X \in M_{2}(B), a \in A\right\} .
$$

Define $\psi: \mathcal{B} \rightarrow \mathcal{B}_{1}$ by $\psi(x)=\left({ }^{x}{ }_{0}\right)$. We claim that $(\mathrm{a}) \psi_{*}: K_{*}(\mathcal{B}) \rightarrow K_{*}\left(\mathcal{B}_{1}\right)$ is injective, and (b) $\psi_{*}=0$. To prove (a) first, note that there is a unitary $U \in \mathbb{L}_{B}(B \oplus B, B)$ such that

$$
U\left(\begin{array}{ll}
\pi(a) & \\
&
\end{array}\right) U^{*}-\pi(a) \in B, a \in A,
$$

because $\pi$ is absorbing. Then $z \mapsto U z U^{*}$ is a $*$-homomorphism $\lambda: \mathcal{B}_{1} \rightarrow \mathcal{B}$. Define $V: B \rightarrow B$ by $V b=U(b, 0)$, and observe that $V$ is adjointable with adjoint $V^{*}: B \rightarrow$ $B$ given by $V^{*} b=p_{1} U^{*} b$, where $p_{1}: B \oplus B \rightarrow B$ is the projection to the first coordinate. $V \in \mathcal{M}(B)$ is an isometry such that $\lambda \circ \psi=\operatorname{Ad} U \circ \psi=\operatorname{Ad} V$ and $V \pi(a) V^{*}=$ $U\left(\begin{array}{ll}\pi(a) & \\ & 0\end{array}\right) U^{*}$. It follows from the last equality and (10) that $V \pi(a)-\pi(a) V \in B$ for all $a \in A$, and then that $x V \in \mathcal{B}$ when $x \in \mathcal{B}$. Therefore $V$ is an isometry in $\mathcal{M}(\mathcal{B})$, and hence $(\operatorname{Ad} V)_{*}=$ id in K-theory. Consequently $\lambda_{*} \circ \psi_{*}=$ id in K-theory, proving (a). To prove (b), observe that $\psi$ is homotopic, via a standard rotation argument, to the $*$-homomorphism $x \mapsto\left({ }^{0}{ }_{x}\right)$, which clearly factors through $\mathcal{M}(B)$. But $K_{*}(\mathcal{M}(B))=0$ since $B$ is stable and hence $\psi_{*}=0$.

By combining Lemma 3.1 with Theorem 3.2 of [25], we conclude that

$$
K_{1}(\mathcal{A})=K K(A, B)
$$

\section{Homotopy invariance}

Let $A$ and $B$ be separable $C^{*}$-algebras, with $B$ stable. By Theorem 2.6, there is an absorbing and saturated $*$-homomorphism $\pi$ : $\operatorname{cone}(A) \rightarrow \mathcal{M}(B)$ such that $\left.\pi\right|_{S A}: S A \rightarrow \mathcal{M}(B)$ is also absorbing and saturated, and a continuous path $p_{t}$, $t \in[0, \infty)$, in $\mathcal{M}(B)$ such that (1)-(5) of Theorem 2.6 hold. We can then define a completely positive asymptotic homomorphism $\lambda=\left(\lambda_{t}\right)_{t \in[1, \infty)}: \operatorname{cone}(A) \rightarrow B$ by $\lambda_{t}(a)=p_{t} \pi(a) p_{t}$. This asymptotic homomorphism will feature in the following theorem. Recall, $[\mathbf{1 0}]$, that $[[\cdot, \cdot]]_{c p}$ denotes the homotopy classes of completely positive asymptotic homomorphisms.

Theorem 4.1. Let $A$ and $B$ be separable $C^{*}$-algebras, $B$ stable. Let $\varphi=\left(\varphi_{t}\right)_{t \in[1, \infty)}$, $\psi=\left(\psi_{t}\right)_{t \in[1, \infty)}: S A \rightarrow B$ be completely positive asymptotic homomorphisms. Then, the following are equivalent: 
(1) $[\varphi]=[\psi]$ in $[[S A, B]]_{c p}$.

(2) There is a completely positive asymptotic homomorphism $\mu=\left(\mu_{t}\right)_{t \in[1, \infty)}$ : $S A \rightarrow B$ and a strictly continuous path $\left\{U_{t}\right\}_{t \in[1, \infty)}$ of unitaries in $\mathcal{M}\left(M_{2}(B)\right)$ such that

$$
\lim _{t \rightarrow \infty} U_{t}\left(\varphi_{t}^{(a)}{ }_{\mu_{t}(a)}\right) U_{t}^{*}-\left(\begin{array}{ll}
\psi_{t}(a) & \\
& \\
& \mu_{t}(a)
\end{array}\right)=0
$$

for all $a \in S A$.

(3) There is a norm-continuous path $\left\{S_{t}\right\}_{t \in[1, \infty)}$ of unitaries in $M_{2}(B)^{+}$and an increasing continuous function $r:[1, \infty) \rightarrow[1, \infty)$ with $\lim _{t \rightarrow \infty} r(t)=\infty$ such that

$$
\lim _{t \rightarrow \infty} S_{t}\left(\begin{array}{ll}
\varphi_{t}(a) & \\
& \lambda_{r(t)}(a)
\end{array}\right) S_{t}^{*}-\left(\begin{array}{ll}
\psi_{t}(a) & \\
& \\
& \lambda_{r(t)}(a)
\end{array}\right)=0
$$

for all $a \in S A$.

Proof. Since $(3) \Rightarrow(2)$ is trivial, it suffices to prove (1) $\Rightarrow(3)$ and (2) $\Rightarrow(1)$. First, $(1) \Rightarrow(3):$ Define $\hat{\varphi}, \hat{\psi}: S A \rightarrow \mathcal{M}\left(C_{0}(0, \infty) \otimes B\right)$ by

$$
(\hat{\varphi}(a) f)(t)=\left\{\begin{array}{ll}
\varphi_{t}(a) f(t), & t \in(1, \infty) \\
t \varphi_{1}(a) f(t), & t \in(0,1]
\end{array}, \quad f \in C_{0}(1, \infty) \otimes B,\right.
$$

and similarly for $\hat{\psi}$. Let $q: \mathcal{M}\left(C_{0}(0, \infty) \otimes B\right) \rightarrow \mathcal{M}\left(C_{0}(0, \infty) \otimes B\right) / C_{0}(0, \infty) \otimes B$ be the quotient map. Then $q \circ \hat{\varphi}$ and $q \circ \hat{\psi}$ define invertible (or semi-split) extensions of $S A$ by $C_{0}(0, \infty) \otimes B$ because $\varphi$ and $\psi$ are completely positive. They define the same element of $\operatorname{Ext}^{-1}\left(S A, C_{0}(0, \infty) \otimes B\right)$ since $\varphi$ and $\psi$ are homotopic as completely positive asymptotic homomorphisms. Such a homotopy gives rise to a diagram of semi-split extensions as in Theorem 3.3.14 of [16], connecting $q \circ \hat{\varphi}$ and $q \circ \hat{\psi}$. Set $\tilde{\pi}=1_{C_{0}(0, \infty)} \otimes \pi$, cf. Lemma 2.3. Since $[q \circ \hat{\varphi}]$ and $[q \circ \hat{\psi}]$ are equal in $\operatorname{Ext}^{-1}\left(S A, C_{0}(0, \infty) \otimes B\right)$ and $\tilde{\pi}$ is absorbing, it follows from Kasparov's theory that there is a unitary $U \in \mathcal{M}\left(M_{2}\left(C_{0}(0, \infty) \otimes B\right)\right)$ such that

$$
U\left(\begin{array}{ll}
\hat{\varphi}(a) & \\
& \tilde{\pi}(a)
\end{array}\right) U^{*}-\left(\begin{array}{cc}
\hat{\psi}(a) & \\
& \tilde{\pi}(a)
\end{array}\right) \in M_{2}\left(C_{0}(0, \infty) \otimes B\right)
$$

for all $a \in S A$. $U$ defines a strictly continuous path, $\left\{U_{t}\right\}_{t \in(0, \infty)}$, of unitaries in $\mathcal{M}\left(M_{2}(B)\right)$ such that

$$
\lim _{t \rightarrow \infty} U_{t}\left(\begin{array}{ll}
\varphi_{t}(a) & \\
& \pi(a)
\end{array}\right) U_{t}^{*}-\left(\begin{array}{ll}
\psi_{t}(a) & \\
& \pi(a)
\end{array}\right)=0
$$

for all $a \in S A$. For each $n \in \mathbb{N}, U_{t}, t \in(0, n]$, defines a unitary $W_{n}$ in $\mathcal{M}\left(M_{2}\left(C_{0}(0, n] \otimes B\right)\right)$. Consider $\tilde{\pi}$ as a $*$-homomorphism $S A \rightarrow \mathcal{M}\left(C_{0}(0, n] \otimes B\right)$ in the obvious way and observe that (12) implies that

$$
W_{n}\left({ }^{0}{ }_{\tilde{\pi}(a)}\right) W_{n}^{*}-\left({ }^{0}{ }_{\tilde{\pi}(a)}\right) \in M_{2}\left(C_{0}(0, n] \otimes B\right)
$$

for all $a \in S A$, i.e. $W_{n}$ is a unitary in

$$
\begin{aligned}
\mathcal{A}_{n}= & \left\{x \in \mathcal{M}\left(M_{2}\left(C_{0}(0, n] \otimes B\right)\right): x\left({ }^{0}{ }_{\tilde{\pi}(a)}\right)\right. \\
& \left.-\left({ }^{0}{ }_{\tilde{\pi}(a)}\right) x \in M_{2}\left(C_{0}(0, n] \otimes B\right), a \in S A\right\} .
\end{aligned}
$$

Note that $\left({ }^{0} \tilde{\pi}\right)$ is absorbing and saturated because $\pi$ has these properties, cf. 
Lemma 2.3. Since $C_{0}(0, n] \otimes B$ is contractible, $K K\left(S A, M_{2}\left(C_{0}(0, n] \otimes B\right)\right)=0$, and we conclude from $(11)$ that $K_{1}\left(\mathcal{A}_{n}\right)=0$. It follows therefore that $\operatorname{diag}\left(W_{n}, 1_{2}, \ldots\right.$, $\left.1_{2}\right)$ is homotopic to $1_{2 k}$ in $M_{k}\left(\mathcal{A}_{n}\right)$ for some $k \in \mathbb{N}$. It is easy to see that since $\tilde{\pi}$ is saturated, we can take $k=2$. Let $E_{n}$ denote the $C^{*}$-subalgebra of $M_{4}\left(\mathcal{M}\left(C_{0}(0, n] \otimes\right.\right.$ $B)$ ) generated by the unit $1_{4}, M_{4}\left(C_{0}(0, n] \otimes B\right)$ and

$$
\left(\begin{array}{lll}
0 & & \\
& \tilde{\pi}(a) & \\
& 0 & \\
& & \tilde{\pi}(a)
\end{array}\right), a \in S A .
$$

It follows from (14) that

$$
\operatorname{Ad}\left(\begin{array}{ll}
W_{n} & \\
& 1_{2}
\end{array}\right)
$$

defines an automorphism $\alpha_{n}$ of $E_{n}$, and the path of unitaries in $M_{2}\left(\mathcal{A}_{n}\right)$ connecting $\left(\begin{array}{ll}W_{n} & \\ & 1_{2}\end{array}\right)$ to $1_{4}$ gives us a uniform norm-continuous path of automorphisms in Aut $E_{n}$ connecting $\alpha_{n}$ to the identity in Aut $E_{n}$. Since $E_{n}$ is separable, it follows from 8.7.8 and 8.6.12 in [22], cf. Proposition 2.15 of [7], that $\alpha_{n}$ is asymptotically inner, i.e. there is a continuous path $V_{s}^{n}, s \in[1, \infty)$, of unitaries in $E_{n}$ such that $\alpha_{n}(x)=$ $\lim _{s \rightarrow \infty} V_{s}^{n} x V_{s}^{n *}$ for all $x \in E_{n}$. For each $t \in(0, n]$, evaluation at $t$ gives us a $*-$ homomorphism ev $: E_{n} \rightarrow \mathcal{M}\left(M_{4}(B)\right)$. Set $V_{s}^{n}(t)=\operatorname{ev}_{t}\left(V_{s}^{n}\right)$. Then each $V_{s}^{n}(t)$ is a unitary in the $C^{*}$-subalgebra $E$ of $M_{4}(\mathcal{M}(B))$ generated by $1_{4}, M_{4}(B)$ and

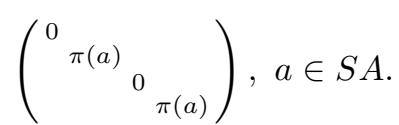

Let $F_{1} \subseteq F_{2} \subseteq F_{3} \subseteq \cdots$ be a sequence of finite subsets with dense union in $S A$. Since

$$
\begin{aligned}
& \lim _{s \rightarrow \infty} \sup _{t \in(0, n]} \| V_{s}^{n}(t)\left(\begin{array}{cccc}
\varphi_{t}(a) & & & \\
& \pi(a) & & \\
& & 0 & \\
& & \pi(a)
\end{array}\right) V_{s}^{n}(t)^{*}
\end{aligned}
$$

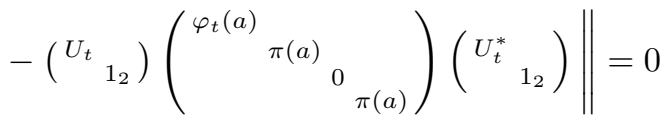

for all $a \in S A$, we can find an $s_{n} \in[1, \infty)$ so big that

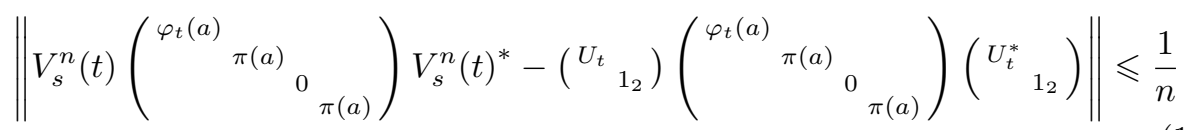

for all $s \geqslant s_{n}$, all $t \in(0, n]$ and all $a \in F_{n}$. For any $b \in B, a \in S A$, we can choose an element $\tilde{x} \in E_{n}$ such that $\operatorname{ev}_{n}(\tilde{x})=x$, where

$$
x=\left(\begin{array}{llll}
b & & & \\
& \pi(a) & & \\
& & 0 & \\
& & & \pi(a)
\end{array}\right) .
$$

Then

$$
\lim _{s \rightarrow \infty} V_{s}^{n}(n) x V_{s}^{n}(n)^{*}=\lim _{s \rightarrow \infty} \operatorname{ev}_{n}\left(V_{s}^{n} \tilde{x} V_{s}^{n *}\right)=\operatorname{ev}_{n}\left(\alpha_{n}(\tilde{x})\right)=\left(\begin{array}{ll}
U_{n} & \\
& 1_{2}
\end{array}\right) x\left(\begin{array}{ll}
U_{n}^{*} & \\
& 1_{2}
\end{array}\right) .
$$

A similar argument shows that $\lim _{s \rightarrow \infty} V_{s}^{n+1}(n) x V_{s}^{n+1}(n)^{*}=\left(\begin{array}{ll}U_{n} & \\ & 1_{2}\end{array}\right) x\left(\begin{array}{ll}U_{n}^{*} & \\ & 1_{2}\end{array}\right)$, 
and hence

$$
\lim _{s \rightarrow \infty} V_{s}^{n+1}(n)^{*} V_{s}^{n}(n) x V_{s}^{n}(n)^{*} V_{s}^{n+1}(n)=x .
$$

To simplify notation, set $\Delta_{s}^{k}=V_{s}^{k+1}(k)^{*} V_{s}^{k}(k)$. It follows from (16) that if we increase $s_{n}$, we can arrange that

$$
\left\|\Delta_{s}^{k}\left(\begin{array}{cccc}
\varphi_{t}(a) & & & \\
& \pi(a) & & \\
& & 0 & \\
& & & \pi(a)
\end{array}\right) \Delta_{s}^{k^{*}}-\left(\begin{array}{cccc}
\varphi_{t}(a) & & & \\
& \pi(a) & & \\
& & 0 & \\
& & & \pi(a)
\end{array}\right)\right\| \leqslant \frac{1}{n^{2}}
$$

for all $a \in F_{n}, t \in(0, n]$, and all $k=2,3, \ldots, n$, when $s \geqslant s_{n}$. Proceeding inductively, we can arrange that $s_{n}<s_{n+1}$ for all $n$. Let $s:[1, \infty) \rightarrow[1, \infty)$ be a continuous increasing function such that $s(n)=s_{n+1}, n=1,2,3, \ldots$ Define a normcontinuous path $W_{t}, t \in[1, \infty)$, in $E$ such that $W_{t}=V_{s(t)}^{2}(t), t \in[1,2]$, and $W_{t}=V_{s(t)}^{k+1}(t) \Delta_{s(t)}^{k} \cdots \Delta_{s(t)}^{3} \Delta_{s(t)}^{2}, \quad t \in[k, k+1], \quad k \geqslant 2$. Let $a \in F_{n}$ and consider $t \in[k, k+1]$, where $k \geqslant n$. Since $s(t) \geqslant s_{k+1}$ and $a \in F_{k+1}$, it follows from (17) that

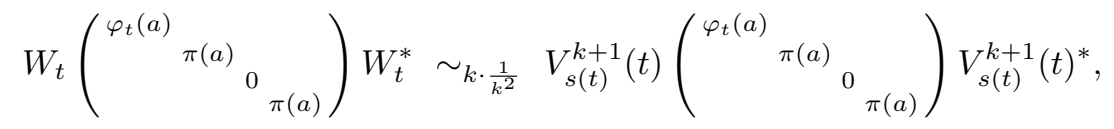

where $\sim_{\delta}$ means that the distance between the two elements is at most $\delta$. Furthermore, it follows from (15) that

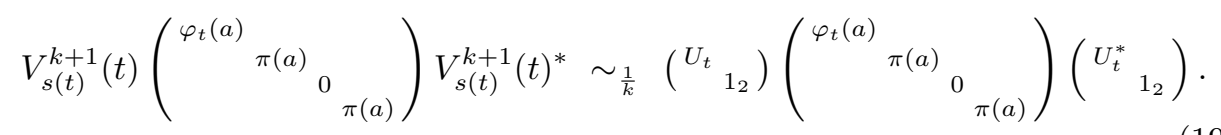

It follows from (19), (18) and (13) that

$$
\lim _{t \rightarrow \infty} W_{t}\left(\begin{array}{cccc}
\varphi_{t}(a) & & & \\
& \pi(a) & & \\
& & 0 & \\
& & & \pi(a)
\end{array}\right) W_{t}^{*}-\left(\begin{array}{cccc}
\psi_{t}(a) & & & \\
& \pi(a) & & \\
& & 0 & \\
& & & \pi(a)
\end{array}\right)=0,
$$

first when $a \in F_{n}$, and then for all $a \in S A$ since $n$ was arbitrary. Being saturated, $\pi$ is unitarily equivalent to $\pi \oplus 0 \oplus \pi$, so there is a unitary $T \in \mathbb{L}_{B}\left(B^{3}, B\right)$ such that $T \operatorname{diag}(\pi(a), 0, \pi(a)) T^{*}=\pi(a), a \in S A$. Set $W=1 \oplus T \in \mathbb{L}_{B}\left(B^{4}, B \oplus\right.$ $B)$. Then $\operatorname{Ad} W(E)=E_{0}$ where $E_{0}$ is the $C^{*}$-subalgebra of $\mathcal{M}\left(M_{2}(B)\right)$ generated by $1_{2}, M_{2}(B)$ and $\left({ }^{0}{ }_{\pi(S A)}\right)$. Set $V_{t}=W W_{t} W^{*} \in E_{0}$, and note that

$$
\lim _{t \rightarrow \infty} V_{t}\left({ }^{\varphi_{t}(a)}{ }_{\pi(a)}\right) V_{t}^{*}-\left(\begin{array}{ll}
\psi_{t}(a) & \\
&
\end{array} \pi_{(a)}\right)=0
$$

for all $a \in S A$. Observe that there is a unique decomposition $V_{t}=\lambda_{t} 1_{2}-a_{t}$, where $\lambda_{t} \in \mathbb{C},\left|\lambda_{t}\right|=1$, and $a_{t} \in\left({ }^{0}{ }_{\pi(S A)}\right)+M_{2}(B)$. By using $\lambda_{t}{ }^{-1} V_{t}$ and $\lambda_{t}^{-1} a_{t}$ instead of $V_{t}$ and $a_{t}$, we can assume that $\lambda_{t}=1$ for all $t$, without violating (21). Set

$$
X_{s, t}=\left(\begin{array}{ll}
{ }^{1} p_{s}
\end{array}\right) V_{t}+\left(\begin{array}{ll}
0 & \\
& 1-p_{s}
\end{array}\right) .
$$

Since $V_{t} \in E_{0}$, it follows from property (2) in Theorem 2.6 that $X_{s, t}=1_{2}$, modulo 


$$
\begin{aligned}
& M_{2}(B) \text {, i.e. } X_{s, t} \in M_{2}(B)^{+} \text {for all } s, t \text {. Furthermore, } \\
& \lim _{s \rightarrow \infty} X_{s, t}^{*} X_{s, t} \\
& =\lim _{s \rightarrow \infty} V_{t}^{*}\left(\begin{array}{cc}
1 & \\
& p_{s}^{2}
\end{array}\right) V_{t}+\left(\begin{array}{ll}
0 & \\
& \left(1-p_{s}\right)^{2}
\end{array}\right)+V_{t}^{*}\left(\begin{array}{cc}
0 & \\
p_{s}-p_{s}^{2}
\end{array}\right)+\left(\begin{array}{cc}
0 & \\
p_{s}-p_{s}^{2}
\end{array}\right) V_{t}
\end{aligned}
$$

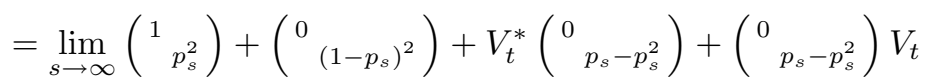

$$
\begin{aligned}
& \text { (using properties (4) and (5) in Theorem 2.6) } \\
& =\lim _{s \rightarrow \infty}\left(\begin{array}{cc}
1 & \\
& p_{s}^{2}
\end{array}\right)+\left(\begin{array}{ll}
0 & \\
& \left(1-p_{s}\right)^{2}
\end{array}\right)+\left(\begin{array}{cc}
0 & \\
& p_{s}-p_{s}^{2}
\end{array}\right)+\left(\begin{array}{cc}
0 & \\
& p_{s}-p_{s}^{2}
\end{array}\right) \\
& \text { (using properties (3) and (4) in Theorem 2.6) } \\
& =1_{2} \text {. }
\end{aligned}
$$

Note that the convergence is uniform for $t$ in compact subsets of $[1, \infty)$. Similarly, we see that $\lim _{s \rightarrow \infty} X_{s, t} X_{s, t}^{*}=1_{2}$, uniformly for $t$ in any compact subset of $[1, \infty)$. It follows that for any $n \in \mathbb{N}$, there is an $m_{n} \in \mathbb{N}$ such that

$$
\sup _{t \in[1, n]}\left\|\left[\left({ }^{1} p_{s}\right), V_{t}\right]\right\|<\frac{1}{n}
$$

and

$$
\sup _{t \in[1, n]}\left(\left\|X_{s, t} X_{s, t}^{*}-1_{2}\right\|+\left\|X_{s, t}^{*} X_{s, t}-1_{2}\right\|\right)<\frac{1}{n}
$$

for all $s \geqslant m_{n}$. We can arrange that $m_{n}<m_{n+1}$ for all $n \in \mathbb{N}$. Define a continuous function $r:[1, \infty) \rightarrow[1, \infty)$ such that $r(n)=m_{n+1}$ and $r$ is linear between $n$ and $n+1$ for all $n$. Then

$$
\begin{aligned}
& \left\|X_{r(t), t}\left(\begin{array}{cc}
\varphi_{t}(a) & \\
& p_{r(t)} \pi(a) p_{r(t)}
\end{array}\right) X_{r(t), t}^{*}-\left(\begin{array}{ll}
\psi_{t}(a) & \\
& p_{r(t)} \pi(a) p_{r(t)}
\end{array}\right)\right\| \\
& \quad \leqslant\left\|V_{t}\left(\begin{array}{cc}
\varphi_{t}(a) & \\
& p_{r(t)} \pi(a) p_{r(t)}
\end{array}\right) V_{t}^{*}-\left(\begin{array}{ll}
\psi_{t}(a) & \\
& p_{r(t)} \pi(a) p_{r(t)}
\end{array}\right)\right\| \\
& \quad \leqslant 2\|a\|\left\|\left[\left(\begin{array}{cc}
1 & \\
& p_{r(t)}
\end{array}\right), V_{t}\right]\right\|+\left\|V_{t}\left(\begin{array}{cc}
\varphi_{t}(a) & \\
& \pi(a)
\end{array}\right) V_{t}^{*}-\left(\begin{array}{ll}
\psi_{t}(a) & \\
& \pi(a)
\end{array}\right)\right\|,
\end{aligned}
$$

which tends to zero as $t$ tends to infinity for all $a \in S A$ by (22) and (21). It follows that

$$
X_{r(t), t}\left(X_{r(t), t}^{*} X_{r(t), t}\right)^{-\frac{1}{2}}
$$

is a norm-continuous path $\left\{S_{t}\right\}_{t \in[1, \infty)}$ of unitaries in $M_{2}(B)^{+}$with the desired properties.

$(2) \Rightarrow(1)$ : By introducing the composition product $\bullet$ for the homotopy classes of completely positive asymptotic homomorphisms, $(2)$ implies that $[\mathcal{U}] \bullet([\varphi]+[\mu])=$ $[\psi]+[\mu]$, where $\mathcal{U}: B \rightarrow B$ is the asymptotic homomorphism $\mathcal{U}_{t}(b)=U_{t} b U_{t}^{*}$. It suffices therefore to show that $[\mathcal{U}]=\left[\mathrm{id}_{B}\right]$ in $[[B, B]]_{c p}$. This is done by connecting $U_{t}$ to 1 via the path $V_{\lambda} U_{t} V_{\lambda}^{*}+\left(1-V_{\lambda} V_{\lambda}^{*}\right)$, where $V_{\lambda}$ is the path of isometries from Lemma 1.3.6 of $[\mathbf{1 6}]$.

In order to apply Theorem 4.1 to asymptotic homomorphisms which are not completely positive, we need a lemma. We let $\mathbb{K}$ denote the $C^{*}$-algebra of compact 
operators on an infinite dimensional separable Hilbert space.

Lemma 4.2. Let $B$ be a separable $C^{*}$-algebra and $D$ a separable $C^{*}$-subalgebra of $C_{b}([1, \infty), B \otimes \mathbb{K}) / C_{0}([1, \infty), B \otimes \mathbb{K})$. There is then a stable separable $C^{*}$-algebra $E$ such that $D \subseteq E \subseteq C_{b}([1, \infty), B \otimes \mathbb{K}) / C_{0}([1, \infty), B \otimes \mathbb{K})$.

Proof. Let $q: C_{b}([1, \infty), B \otimes \mathbb{K}) \rightarrow C_{b}([1, \infty), B \otimes \mathbb{K}) / C_{0}([1, \infty), B \otimes \mathbb{K})$ be the quotient map.

Observation 4.3. Let $f \in C_{b}([1, \infty), B \otimes \mathbb{K})$ be a positive element. There is then an element $z \in C_{b}([1, \infty), B \otimes \mathbb{K}) / C_{0}([1, \infty), B \otimes \mathbb{K})$ such that $z^{*} z=q(f)$ and $z z^{*} q(f)$ $=0$.

To prove this observation, construct first a $g \in C_{b}([1, \infty), B \otimes \mathbb{K})$ such that $\lim _{t \rightarrow \infty} f(t)-g(t)=0$, and $g$ has the following property: For each $n \in \mathbb{N}$, there is an $m_{n} \in \mathbb{N}$ such that $g(t) \in B \otimes M_{m_{n}}(\mathbb{C}) \subseteq B \otimes \mathbb{K}, t \in[1, n]$. Then construct, recursively, elements $z_{n} \in C([1, n], B \otimes \mathbb{K})$ such that $z_{n}^{*}(t) z_{n}(t)=g(t), z_{n}(t) z_{n}^{*}(t) g(t)=$ $0, t \in[1, n]$, and $z_{n}^{*}(t) z_{i}(t)=0, t \in[1, i], i \leqslant n-1$. Choose finally a partition of unity $\left\{h_{i}\right\}_{i=2}^{\infty} \subseteq C_{b}[1, \infty)$ such that $\operatorname{supp} h_{i} \subseteq[1, i]$ and set $z=q\left(z_{0}\right)$ where $z_{0}(t)=$ $\sum_{i=2}^{\infty} \sqrt{h_{i}(t)} z_{i}(t)$.

It follows from Observation 4.3 that we can find a sequence $D \subseteq D_{1} \subseteq D_{2} \subseteq \ldots$ of separable $C^{*}$-subalgebras of $C_{b}([1, \infty), B \otimes \mathbb{K}) / C_{0}([1, \infty), B \otimes \mathbb{K})$ and for each $n$, have a dense sequence $\left\{g_{1}, g_{2}, \ldots\right\}$ in the positive part of $D_{n}$ and elements $\left\{v_{1}, v_{2}, \ldots\right\}$ in $D_{n+1}$ such that $v_{k}^{*} v_{k}=g_{k}$ and $v_{k} v_{k}^{*} g_{k}=0$ for all $k$. Set $E=\overline{\bigcup_{n=1}^{\infty} D_{n}}$ which is a separable $C^{*}$-subalgebra of $C_{b}([1, \infty), B \otimes \mathbb{K}) / C_{0}([1, \infty), B \otimes \mathbb{K})$ containing $D$. If $a \in E$ is a positive element and $\epsilon>0$, there are elements $b, x \in E, b \geqslant 0$, such that $\|a-b\|<\epsilon, x^{*} x=b$ and $x x^{*} b=0$. By Proposition 2.2 and Theorem 2.1 of $[\mathbf{9}]$, we conclude that $E$ is stable.

Theorem 4.4. Let $A$ and $B$ be separable $C^{*}$-algebras, $B$ stable. Let $\varphi=\left(\varphi_{t}\right)_{t \in[1, \infty)}$, $\psi=\left(\psi_{t}\right)_{t \in[1, \infty)}: S A \rightarrow B$ be asymptotic homomorphisms. Then the following are equivalent:

(1) $[\varphi]=[\psi]$ in $[[S A, B]]$.

(2) There is an asymptotic homomorphism $\nu=\left(\nu_{t}\right)_{t \in[1, \infty)}: \operatorname{cone}(A) \rightarrow B$ and a norm-continuous path $\left\{U_{t}\right\}_{t \in[1, \infty)}$ of unitaries in $M_{2}(B)^{+}$such that

$$
\lim _{t \rightarrow \infty} U_{t}\left(\begin{array}{ll}
\varphi_{t}(a) & \\
& \nu_{t}(a)
\end{array}\right) U_{t}^{*}-\left(\begin{array}{ll}
\psi_{t}(a) & \\
& \nu_{t}(a)
\end{array}\right)=0
$$

for all $a \in S A$.

Proof. The implication $(2) \Rightarrow(1)$ is proved in the same way as the corresponding implication in the proof of Theorem 4.1. We prove $(1) \Rightarrow(2)$ : Define $\tilde{\varphi}, \tilde{\psi}: S A \rightarrow$ $C_{b}([1, \infty), B)$ such that

$$
\tilde{\varphi}(a)(t)=\varphi_{t}(a)
$$

and similarly for $\psi$. Let $q_{B}: C_{b}([1, \infty), B) \rightarrow C_{b}([1, \infty), B) / C_{0}([1, \infty), B)$ be quotient map and note that $q_{B} \circ \tilde{\varphi}$ and $q_{B} \circ \tilde{\psi}$ are both $*$-homomorphisms. It follows from the equivalence between (1) and (2) in Theorem 1.1, which was established in 
Theorem 3.4 of $[\mathbf{2 0}]$, that $q_{B} \circ \tilde{\varphi}$ and $q_{B} \circ \tilde{\psi}$ are homotopic as $*$-homomorphisms. Since $A$ is separable, we conclude from Lemma 4.2 that there is a separable and stable $C^{*}$-subalgebra $D$ of $C_{b}([1, \infty), B) / C_{0}([1, \infty), B)$ such that $q_{B} \circ \tilde{\varphi}, q_{B} \circ \tilde{\psi}$ take values in $D$ and are homotopic in $\operatorname{Hom}(S A, D)$. By Theorem 4.1, there is a completely positive asymptotic homomorphism $\mu$ : $\operatorname{cone}(A) \rightarrow D$ and a normcontinuous path $\left\{S_{t}\right\}_{t \in[1, \infty)}$ in $M_{2}(D)^{+}$such that

$$
\lim _{t \rightarrow \infty} S_{t}\left(\begin{array}{ll}
q_{B} \circ \tilde{\varphi}(a) & \\
& \mu_{t}(a)
\end{array}\right) S_{t}^{*}-\left(\begin{array}{ll}
q_{B} \circ \tilde{\psi}(a) & \\
& \mu_{t}(a)
\end{array}\right)=0
$$

for all $a \in S A$. Let $\chi$ be a continuous right-inverse for the quotient map $q_{B}: C_{b}([1, \infty), B) \rightarrow C_{b}([1, \infty), B) / C_{0}([1, \infty), B)$. Lift $S$ to a norm-continuous path $W=\left\{W_{t}\right\}$ of unitaries in $C_{b}\left([1, \infty), M_{2}(B)^{+}\right)$. (If necessary, the explicit construction of such a lift can be found in Lemma 3.3 of $[\mathbf{2 8}]$.) If $r:[1, \infty) \rightarrow[1, \infty)$ is a continuous and sufficiently slowly increasing function with $\lim _{t \rightarrow \infty} r(t)=\infty$, then $\nu=\left(\chi \circ \mu_{r(t)}(\cdot)(t)\right)_{t \in[1, \infty)}$ is an asymptotic homomorphism $\nu: \operatorname{cone}(A) \rightarrow B$ such that

$$
\lim _{t \rightarrow \infty} W_{r(t)}(t)\left(\begin{array}{lll}
\varphi_{t}(a) & \\
& \nu_{t}(a)
\end{array}\right) W_{r(t)}(t)^{*}-\left(\begin{array}{ll}
\psi_{t}(a) & \\
& \nu_{t}(a)
\end{array}\right)=0
$$

for all $a \in S A$. Since $t \mapsto W_{r(t)}(t)$ is norm-continuous, we are done.

Note that the last result improves on Theorem 3.4 of $[\mathbf{2 0}]$ in two ways: The asymptotic homomorphism $\nu$ is defined on cone $(A)$, not only on $S A$, and the path of unitaries comes from $M_{2}(B)^{+}$rather than $\mathcal{M}\left(M_{2}(B)\right)$.

\section{A description of E-theory in terms of KK-theory}

In this section, we will use the results of the previous sections to show that

$$
E(A, B) \simeq K K\left(A, C_{b}([1, \infty), B \otimes \mathbb{K}) / C_{0}([1, \infty), B \otimes \mathbb{K})\right),
$$

when $A$ and $B$ are separable $C^{*}$-algebras. Since the second variable of the KKfunctor in this statement is not even $\sigma$-unital, we must point out that we use the following definition regarding the KK-theory of a non-separable $C^{*}$-algebra $D$ :

$$
K K(A, D)=\lim _{T} K K(A, T),
$$

where the limit is taken over the net of separable $C^{*}$-subalgebras $T$ of $D$ ordered by inclusion.

Assume that $B$ is stable. It follows from Theorem 1.1 that if two asymptotic homomorphisms, $\varphi=\left(\varphi_{t}\right)_{t \in[1, \infty)}, \psi=\left(\psi_{t}\right)_{t \in[1, \infty)}: S A \rightarrow B$, represent the same element in $[[S A, B]]$, the two $*$-homomorphisms, $q_{B} \circ \tilde{\varphi}, q_{B} \circ \tilde{\psi}: S A \rightarrow C_{b}([1, \infty), B) /$ $C_{0}([1, \infty), B)$, which they define are homotopic. In particular, it follows that the recipe $[\varphi] \mapsto\left[q_{B} \circ \tilde{\varphi}\right]$ defines a homomorphism $\mathbf{\Phi}:[[S A, B]] \rightarrow K K\left(S A, C_{b}([1, \infty)\right.$, $\left.B) / C_{0}([1, \infty), B)\right)$.

Theorem 5.1. Let $A$ and $B$ be separable $C^{*}$-algebras with $B$ is stable. Then $\mathbf{\Phi}$ : $[[S A, B]] \rightarrow K K\left(S A, C_{b}([1, \infty), B) / C_{0}([1, \infty), B)\right)$ is an isomorphism. 
Proof. Injectivity: If $\boldsymbol{\Phi}[\varphi]=\boldsymbol{\Phi}[\psi]$, it follows that there is a separable $C^{*}$-subalgebra $D$ of $C_{b}([1, \infty), B) / C_{0}([1, \infty), B)$ such that $q_{B} \circ \tilde{\varphi}(S A) \cup q_{B} \circ \tilde{\psi}(S A) \subseteq D$ and $\left[q_{B} \circ \tilde{\varphi}\right]=\left[q_{B} \circ \tilde{\psi}\right]$ in $K K(S A, D)$. By Lemma 4.2, we may assume that $D$ is stable. As pointed out in $[\mathbf{1 9}]$, it follows from $[\mathbf{1 0}]$ and $[\mathbf{6}]$ that $K K(S A, D)=[[S A, D]]_{c p}$. So, we conclude from Theorem 4.1 that there is a norm-continuous path $\left\{V_{t}\right\}_{t \in[1, \infty)}$ of unitaries in $M_{2}(D)^{+}$and a completely positive asymptotic homomorphism $\mu: S A \rightarrow D$ such that

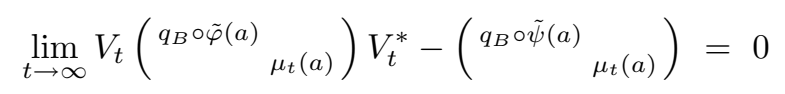

for all $a \in S A$. As in the proof of $(1) \Rightarrow(2)$ in Theorem 4.4, we can 'lift' this to get a path of unitaries $\left\{U_{t}\right\}_{t \in[1, \infty)} \subseteq M_{2}(B)^{+}$and an asymptotic homomorphism $\nu: S A \rightarrow B$ such that

$$
\lim _{t \rightarrow \infty} U_{t}\left(\begin{array}{ll}
\varphi_{t}(a) & \\
& \nu_{t}(a)
\end{array}\right) U_{t}^{*}-\left(\begin{array}{ll}
\psi_{t}(a) & \\
& \nu_{t}(a)
\end{array}\right)=0
$$

for all $a \in S A$. It follows that $[\varphi]=[\psi]$ in $[[S A, B]]$.

Surjectivity: We must show that each element of $K K\left(S A, C_{b}([1, \infty), B) /\right.$ $\left.C_{0}([1, \infty), B)\right)$ is represented by a $*$-homomorphism. To this end, it suffices by Lemma 4.2 to consider a separable stable $C^{*}$-subalgebra $D \subseteq C_{b}([1, \infty), B) /$ $C_{0}([1, \infty), B)$ and show that for any element $x \in K K(S A, D)$ there is a separable $C^{*}$-algebra $D_{1}$ such that $D \subseteq D_{1} \subseteq C_{b}([1, \infty), B) / C_{0}([1, \infty), B)$ and such that the image of $x$ in $K K\left(S A, D_{1}\right)$ is represented by a *-homomorphism. To this end, we use the Cuntz-Higson picture of KK-theory. There is then a pair $\varphi_{1}, \varphi_{2}: S A \rightarrow \mathcal{M}(D)$ of $*$-homomorphisms such that $\varphi_{1}(a)-\varphi_{2}(a) \in D$ for all $a \in S A$ and $\left[\varphi_{1}, \varphi_{2}\right]=x$ in $K K(S A, D)$. Let $\pi: S A \rightarrow \mathcal{M}(D)$ be an absorbing and saturated $*$-homomorphism, and $\left\{p_{t}\right\}_{t \in[1, \infty)}$ a continuous path of elements in $\mathcal{M}(D)$ such that (1)-(5) of Theorem 2.6 hold. Since $\pi$ is absorbing, there is a unitary $W \in \mathcal{M}(D)$ such that $W\left(\pi(a) \oplus \varphi_{1}(a)\right) W^{*}-\pi(a) \in D$ for all $a \in S A$. If we substitute $\operatorname{Ad} W \circ\left(\pi \oplus \varphi_{k}\right)$ for $\varphi_{k}, k=1,2$, we will still have that $x=\left[\varphi_{1}, \varphi_{2}\right]$, and in addition $p_{t} \varphi_{i}(a) \in D$ for all $t \in[1, \infty), i=1,2, \quad \lim _{t \rightarrow \infty} p_{t} d=d$ and $\lim _{t \rightarrow \infty} p_{t} \varphi_{i}(a)-\varphi_{i}(a) p_{t}=0, \lim _{t \rightarrow \infty}\left(p_{t}^{2}-p_{t}\right) \varphi_{i}(a)=0, i=1,2$, for all $d \in D$ and all $a \in S A$. Let $\chi: C_{b}([1, \infty), B) / C_{0}([1, \infty), B) \rightarrow C_{b}([1, \infty), B)$ be a continuous right-inverse for $q_{B}$. Let $\left\{z_{i}\right\}$ and $\left\{d_{i}\right\}$ be dense sequences in $S A$ and $D$, respectively. Set $g_{i}=\chi\left(d_{i}\right)$. For each $n \in \mathbb{N}$, there is a $N_{n} \in \mathbb{N}$ so large that

$$
\begin{aligned}
& \left\|\chi\left(p_{t} \varphi_{l}\left(z_{j_{1}}\right) p_{t}\right)(s)+\chi\left(p_{t} \varphi_{l}\left(z_{j_{2}}\right) p_{t}\right)(s)-\chi\left(p_{t} \varphi_{l}\left(z_{j_{1}}+z_{j_{2}}\right) p_{t}\right)(s)\right\| \leqslant \frac{1}{n} \\
& \left\|\chi\left(p_{t} \varphi_{l}\left(z_{j_{1}}\right) p_{t}\right)(s) \chi\left(p_{t} \varphi_{l}\left(z_{j_{2}}\right) p_{t}\right)(s)-\chi\left(p_{t} \varphi_{l}\left(z_{j_{1}} z_{j_{2}}\right) p_{t}\right)(s)\right\| \\
& \leqslant\left\|p_{t} \varphi_{l}\left(z_{j_{1}}\right) p_{t}^{2} \varphi_{l}\left(z_{j_{2}}\right) p_{t}-p_{t} \varphi_{l}\left(z_{j_{1}} z_{j_{2}}\right) p_{t}\right\|+\frac{1}{n}, \\
& \left\|\chi\left(p_{t} \varphi_{l}\left(z_{j_{1}}\right) p_{t}\right)(s)^{*}-\chi\left(p_{t} \varphi_{l}\left(z_{j_{1}}^{*}\right) p_{t}\right)(s)\right\| \leqslant \frac{1}{n}
\end{aligned}
$$




$$
\begin{aligned}
& \left\|\chi\left(p_{t} \varphi_{l}\left(z_{j_{1}}\right) p_{t}\right)(s) g_{k}(s)-\chi\left(\varphi_{l}\left(z_{j_{1}}\right)\right)(s) g_{k}(s)\right\| \leqslant\left\|p_{t} \varphi_{l}\left(z_{j_{1}}\right) p_{t} d_{k}-\varphi_{l}\left(z_{j_{1}}\right) d_{k}\right\|+\frac{1}{n} \\
& \left\|\chi\left(p_{t} \varphi_{1}\left(z_{j_{1}}\right) p_{t}\right)(s)-\chi\left(p_{t} \varphi_{2}\left(z_{j_{1}}\right) p_{t}\right)(s)-\chi\left(\varphi_{1}\left(z_{j_{1}}\right)-\varphi_{2}\left(z_{j_{1}}\right)\right)(s)\right\| \\
& \leqslant\left\|p_{t} \varphi_{1}\left(z_{j_{1}}\right) p_{t}-p_{t} \varphi_{2}\left(z_{j_{1}}\right) p_{t}-\left(\varphi_{1}\left(z_{j_{1}}\right)-\varphi_{2}\left(z_{j_{1}}\right)\right)\right\|+\frac{1}{n}
\end{aligned}
$$

for all $j_{1}, j_{2}, k \in\{1,2, \ldots, n\}$, all $t \in[1, n]$ and all $s \geqslant N_{n}, l=1,2$. We may assume that $N_{n}<N_{n+1}$ for all $n$. Let $r:[1, \infty) \rightarrow[1, \infty)$ be a continuous increasing function such that $r\left(N_{n}\right)=n-1, n \geqslant 2$. It follows from the first three inequalities above that $\alpha_{t}^{l}(z)=\chi\left(p_{r(t)} \varphi_{l}(z) p_{r(t)}\right)(t)$ defines an asymptotic homomorphism $\left\{\alpha_{t}^{l}\right\}_{t \in[1, \infty)}: S A \rightarrow B, l=1,2$. Let $\alpha_{l}: S A \rightarrow C_{b}([1, \infty), B) / C_{0}([1, \infty), B)$ be the $*$-homomorphism defined from $\left\{\alpha_{t}^{l}\right\}_{t \in[1, \infty)}$. It follows from the fourth inequality above that

$$
\alpha_{l}(z) b=\varphi_{l}(z) b
$$

for all $z \in S A, b \in D, l=1,2$, and from the last that

$$
\varphi_{1}(z)-\varphi_{2}(z)=\alpha_{1}(z)-\alpha_{2}(z),
$$

$z \in S A$. By Lemma 4.2, we can find a separable stable $C^{*}$-subalgebra $D_{1}$ of $C_{b}([1, \infty), B) / C_{0}([1, \infty), B)$ such that $D \cup \alpha_{1}(S A) \cup \alpha_{2}(S A) \subseteq D_{1}$. Then the image of $x$ in $K K\left(S A, D_{1}\right)$ is represented by the pair $\left(\alpha_{1}, \alpha_{2}\right)$. This is best checked via Cuntz' picture of KK, [5]. Indeed, (23) and (24) imply that the $*$-homomorphisms $q(S A) \rightarrow D_{1}$ coming from $\left(\varphi_{1}, \varphi_{2}\right)$ and $\left(\alpha_{1}, \alpha_{2}\right)$ are identical. Since $\alpha_{1}$ and $\alpha_{2}$ both takes values in $D_{1}$, a standard homotopy argument shows that $\left(\alpha_{1}, \alpha_{2}\right)$ defines the same element of $K K\left(S A, D_{1}\right)$ as the $*$-homomorphism $\alpha_{1} \oplus \alpha_{2} \circ \gamma$ where $\gamma: S A \rightarrow$ $S A$ is the automorphism $\gamma(f)(t)=f(1-t), t \in[0,1]$.

\section{An example}

It is natural to ask to what extent the main results of this paper depend on having a suspended $C^{*}$-algebra as domain algebra. Let us therefore in conclusion show by example that the implications $(1) \Rightarrow(2)$ of Theorem 1.1 and $(1) \Rightarrow(2)$ of Theorem 4.1 can fail when this is not the case.

Example 6.1. In this example, we exhibit an asymptotic homomorphism $\varphi^{0}=$ $\left(\varphi_{t}^{0}\right)_{t \in[1, \infty)}: C(\mathbb{T}) \rightarrow C(\mathbb{T}) \otimes \mathbb{K}$ consisting of $*$-homomorphisms and a $*$-homomor$\operatorname{phism} \varphi^{1}: C(\mathbb{T}) \rightarrow C(\mathbb{T}) \otimes \mathbb{K}$ such that $\left[\varphi^{0}\right]=\left[\varphi^{1}\right]$ in $[[C(\mathbb{T}), C(\mathbb{T}) \otimes \mathbb{K}]]_{c p}$, but such that $\varphi^{0}$ and $\varphi^{1}$ are not equi-homotopic as asymptotic homomorphisms, i.e. there is no homotopy of asymptotic homomorphisms between $\varphi^{0}$ and $\varphi^{1}$ with the equicontinuity property described in (2) of Theorem 1.1.

We identify $C(\mathbb{T})$ with $\{f \in C[0,1]: f(0)=f(1)\}$ and $C(\mathbb{T}) \otimes \mathbb{K}$ with $\{f \in$ $C([0,1], \mathbb{K}): f(0)=f(1)\}$, and we denote by $\left\{e_{i j}\right\}_{i, j=1}^{\infty}$ the standard system of matrix units in $\mathbb{K}$. Let $h_{t}:[0,1] \rightarrow \mathbb{R}$ be the continuous function which is affine on $\left[0, \frac{1}{2}\right]$ and $\left[\frac{1}{2}, 1\right]$ such that $h_{t}(0)=h_{t}(1)=0$ and $h_{t}\left(\frac{1}{2}\right)=t$. Define $\varphi_{t}^{0}: C(\mathbb{T}) \rightarrow$ 
$C(\mathbb{T}) \otimes \mathbb{K}$ by

$$
\varphi_{t}^{0}(f)(x)=f\left(e^{2 \pi i h_{t}(x)}\right) e_{11}, x \in[0,1] .
$$

Set $\varphi^{1}=\varphi_{0}^{0}$. Define $\boldsymbol{\Phi}=\left(\boldsymbol{\Phi}_{t}\right)_{t \in[1, \infty)}: C(\mathbb{T}) \rightarrow C[0,1] \otimes C(\mathbb{T}) \otimes \mathbb{K}$ by

$$
\boldsymbol{\Phi}_{t}(f)(s, x)=f\left(e^{2 \pi i s h_{t}(x)}\right) e_{11}, s \in[0,1], x \in[0,1] .
$$

Then $\boldsymbol{\Phi}$ is a continuous path of $*$-homomorphisms $C(\mathbb{T}) \rightarrow C[0,1] \otimes C(\mathbb{T}) \otimes \mathbb{K}$, and hence in particular an asymptotic homomorphism, showing that $\left[\varphi^{0}\right]=\left[\varphi^{1}\right]$ in $[[C(\mathbb{T}), C(\mathbb{T}) \otimes \mathbb{K}]]_{c p}$.

To see that $\varphi^{0}$ and $\varphi^{1}$ cannot be equi-homotopic, assume that $\boldsymbol{\Psi}^{\lambda}, \lambda \in[0,1]$, is an equi-homotopy with $\boldsymbol{\Psi}^{0}=\varphi^{0}$ and $\boldsymbol{\Psi}^{1}=\varphi^{1}$. Since $\lim _{t \rightarrow \infty} \sup _{\lambda \in[0,1]} \| \boldsymbol{\Psi}_{t}^{\lambda}(1)-$ $\boldsymbol{\Psi}_{t}^{\lambda}(1)^{*}\left\|=\lim _{t \rightarrow \infty} \sup _{\lambda \in[0,1]}\right\| \boldsymbol{\Psi}_{t}^{\lambda}(1)^{2}-\boldsymbol{\Psi}_{t}^{\lambda}(1) \|=0$, the spectral projection of $1 / 2$ $\left(\boldsymbol{\Psi}_{t}^{\lambda}(1)+\boldsymbol{\Psi}_{t}^{\lambda}(1)^{*}\right)$ corresponding to the interval [1/2,3/2], will give us a continuous family of projections, $p_{\lambda, t} \in C(\mathbb{T}) \otimes \mathbb{K}$, such that

$$
\lim _{t \rightarrow \infty} \sup _{\lambda \in[0,1]}\left\|\Psi_{t}^{\lambda}(1)-p_{\lambda, t}\right\|=0 .
$$

Since $p_{0, t}=1_{C(\mathbb{T})} \otimes e_{11}$ for all $t$ large enough, we see that $p_{s, t}$ is a rank 1 projection for all $s, t$. Let $z$ be the identity function on $\mathbb{T}$. For $t$ large enough, $p_{\lambda, t} \boldsymbol{\Psi}_{t}^{\lambda}(z) p_{\lambda, t}$ will be invertible in $p_{\lambda, t}(C(\mathbb{T}) \otimes \mathbb{K}) p_{\lambda, t}$ for all $\lambda$, and the unitary from its polardecomposition will give us a continuous family of functions $g_{\lambda, t} \in C(\mathbb{T}, \mathbb{T})$ such that

$$
\lim _{t \rightarrow \infty} \sup _{\lambda \in[0,1]}\left\|\Psi_{t}^{\lambda}(z)-g_{\lambda, t} p_{\lambda, t}\right\|=0 .
$$

It follows from (25) and (26) that there is a $K \geqslant 1$ such that

$$
\begin{aligned}
& \left\|g(\lambda, t)-g\left(\lambda^{\prime}, t\right)\right\|_{C(\mathbb{T})}=\left\|g(\lambda, t) p_{(\lambda, t)}-g\left(\lambda^{\prime}, t\right) p_{(\lambda, t)}\right\|_{C(\mathbb{T}) \otimes \mathbb{K}} \\
& \leqslant\left\|g(\lambda, t) p_{(\lambda, t)}-g\left(\lambda^{\prime}, t\right) p_{\left(\lambda^{\prime}, t\right)}\right\|_{C(\mathbb{T}) \otimes \mathbb{K}}+\left\|p_{\left(\lambda^{\prime}, t\right)}-p_{(\lambda, t)}\right\|_{C(\mathbb{T}) \otimes \mathbb{K}} \\
& \leqslant\left\|\boldsymbol{\Psi}_{t}^{\lambda}(z)-\mathbf{\Psi}_{t}^{\lambda^{\prime}}(z)\right\|+\left\|\mathbf{\Psi}_{t}^{\lambda}(1)-\mathbf{\Psi}_{t}^{\lambda^{\prime}}(1)\right\|+1 / 2
\end{aligned}
$$

for all $\lambda, \lambda^{\prime} \in[0,1]$ and all $t \geqslant K$. Combined with the equi-continuity of $\boldsymbol{\Psi}$, this shows that there exists a $\delta>0$ such that

$$
\lambda, \lambda^{\prime} \in[0,1],\left|\lambda-\lambda^{\prime}\right|<\delta, t \geqslant K \Rightarrow\left\|g(\lambda, t)-g\left(\lambda^{\prime}, t\right)\right\|<1 .
$$

By increasing $K$ if necessary, we may assume that $g(0, t)(x)=e^{2 \pi i h_{t}(x)}, g(1, t)(x)=$ $1, x \in[0,1], t \geqslant K$. It follows from (27) that there is a finite set of continuous realvalued functions $\tau_{1}, \tau_{2}, \cdots, \tau_{m}:[K, \infty) \times[0,1] \rightarrow \mathbb{R}$ such that $\left|\tau_{i}(t, x)\right|<1$, and

$$
e^{2 \pi i h_{t}(x)}=e^{2 \pi i \tau_{1}(t, x)} e^{2 \pi i \tau_{2}(t, x)} \cdots e^{2 \pi i \tau_{m}(t, x)}
$$

for all $x \in[0,1]$ and all $t \geqslant K$. Hence, $h_{t}(x)-\sum_{i=1}^{m} \tau_{i}(t, x) \in \mathbb{Z}$ for all $x$ and all $t \geqslant K$, and by continuity, we conclude that

$$
h_{t}(x)=\sum_{i=1}^{m} \tau_{i}(t, x)+c, t \geqslant K, x \in[0,1]
$$

for some constant $c \in \mathbb{Z}$. Since $\left|\sum_{i=1}^{m} \tau_{i}(t, x)\right| \leqslant m$ for all $t \geqslant K$ and all $x \in[0,1]$, this contradicts the fact that $\lim _{t \rightarrow \infty} h_{t}\left(\frac{1}{2}\right)=\infty$. 
Let us also show that there does not exist an asymptotic homomorphism $\lambda=$ $\left(\lambda_{t}\right)_{t \in[1, \infty)}: C(\mathbb{T}) \rightarrow C(\mathbb{T}) \otimes \mathbb{K}$ and a strictly continuous path $\left\{W_{t}\right\}_{t \in[1, \infty)}$ of unitaries in $M_{2}(\mathcal{M}(C(\mathbb{T}) \otimes \mathbb{K}))$ such that

$$
\lim _{t \rightarrow \infty} W_{t}\left(\begin{array}{cc}
\varphi_{t}^{0}(a) & \\
& \lambda_{t}(a)
\end{array}\right) W_{t}^{*}-\left(\begin{array}{cc}
\varphi_{t}^{1}(a) & \\
& \lambda_{t}(a)
\end{array}\right)=0
$$

for all $a \in C(\mathbb{T})$. If there did, $\lambda_{t}(1)$ would be asymptotic to a projection of constant rank, say $m$, and there would be an asymptotic homomorphism $\lambda^{\prime}: C(\mathbb{T}) \rightarrow C(\mathbb{T}) \otimes$ $M_{m}(\mathbb{C}) \subseteq C(\mathbb{T}) \otimes \mathbb{K}$ such that $\lambda_{t}^{\prime}(1)=\sum_{i=1}^{m} e_{i i}$ and a norm-continuous path of unitaries $\left\{S_{t}\right\}_{t \in[1, \infty)}$ in $\mathcal{M}(C(\mathbb{T}) \otimes \mathbb{K})$ such that $\lim _{t \rightarrow \infty} S_{t} \lambda_{t}^{\prime}(a) S_{t}^{*}-\lambda_{t}(a)=0$ for all $a$. So, by working with $\lambda^{\prime}$ in place of $\lambda$ and

$$
W_{t}^{\prime}=\left(\begin{array}{ll}
{ }^{1} & \\
& S_{t}
\end{array}\right) W_{t}\left({ }^{1} S_{t}^{*}\right)
$$

in place of $W$, we may as well assume that $\lambda_{t}(1)=\sum_{i=1}^{m} e_{i i}$ for all $t$. Then

$$
\lim _{t \rightarrow \infty}\left\|W_{t} p-p W_{t}\right\|=0,
$$

where

$$
p=\left(\begin{array}{lll}
\varphi_{t}^{0}(1) & \\
& \lambda_{t}(1)
\end{array}\right)=\left(\begin{array}{lll}
e_{11} & & \\
& \sum_{i=1}^{m} e_{i i}
\end{array}\right),
$$

and we can therefore substitute $W$ with a norm-continuous path of unitaries in

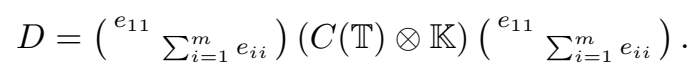

Since

$$
\lim _{t \rightarrow \infty}\left\|W_{t}\left(\begin{array}{cc}
\varphi_{t}^{0}(z) & \\
& \lambda_{t}(z)
\end{array}\right) W_{t}^{*}-\left(\begin{array}{cc}
\varphi_{t}^{1}(z) & \\
& \lambda_{t}(z)
\end{array}\right)\right\|=0,
$$

we would get a $K>1$ and a norm-continuous path $a_{t}=a_{t}^{*}, t \in[K, \infty)$, of elements in $D$ such that $\left\|a_{t}\right\| \leqslant 1$ and

$$
W_{t}\left(\begin{array}{ll}
\varphi_{t}^{0}(z) & \\
& \lambda_{t}(z)
\end{array}\right) W_{t}^{*}=e^{2 \pi i a_{t}}\left(\begin{array}{lll}
\varphi_{t}^{1}(z) & \\
& & \\
& & \\
& & \\
& & \\
&
\end{array}\right)
$$

for all $t \geqslant K$. Since $D$ is a copy of $C(\mathbb{T}) \otimes M_{m+1}(\mathbb{C})$, we can take determinants in $M_{m+1}(\mathbb{C})$ to conclude from $(28)$ that $e^{2 \pi i \operatorname{Tr}\left(a_{t}(s)\right)}=e^{2 \pi i h_{t}(s)}$ for all $s \in[0,1]$ and all $t \geqslant K$. This would imply that

$$
\operatorname{Tr}\left(a_{t}(s)\right)-h_{t}(s)=c, \quad t \geqslant K, s \in[0,1],
$$

for some integer constant $c$. Since $\left|\operatorname{Tr}\left(a_{t}(s)\right)\right| \leqslant m+1$ for all $t, s$, this contradicts again that $\lim _{t \rightarrow \infty} h_{t}(1 / 2)=\infty$.

\section{Acknowledgements}

Some of the results of the present paper have non-empty overlap with results obtained by Dadarlat and Eilers in [7]. One of the key ideas in the proof of the main results - the one which produces an approximate inner automorphism out of a trivial KK-element - I learned from their work. This idea was first introduced by Huaxin Lin in [17]. I am grateful to all three, Dadarlat, Eilers and Lin, for keeping me informed about their work. 


\section{References}

[1] C. Anantharaman-Delaroche, Classification des $C^{*}$-algèbres purement infinies nucléaires (d'apres E. Kirchberg), Astérisque, 241 (1997), pp. 7-27.

[2] W. Arveson, Notes on extensions, Duke Math. J., 44 (1977), pp. 329-355.

[3] L. Brown, R. Douglas and P. Fillmore, Extensions of $C^{*}$-algebras and $K$ homology, Ann. of Math., 105 (1977), pp. 265-324.

[4] A. Connes and N. Higson, Deformations, morphisms asymptotiques et $K$ theorie bivariante, C.R. Acad. Sci. Paris, Sér. I Math., 313 (1990), pp. 101106.

[5] J. Cuntz, A New Look at KK-theory, K-theory, 1 (1987), pp. 31-51.

[6] M. Dadarlat and T. Loring, K-homology, Asymptotic Representations, and Unsuspended E-theory, J. Func. Analysis, 126 (1994), pp. 367-383.

[7] M. Dadarlat and S. Eilers, Asymptotic unitary equivalence in KK-theory, $K$ Theory, 23(4) (2001), pp. 305-322.

[8] N. Higson, Algebraic $K$-theory of stable $C^{*}$-algebras, Advances in Math., 67 (1988), pp. 1-140.

[9] J. Hjelmborg and M. Rørdam, On stability of $C^{*}$-algebras, J. Funct. Anal., 155 (1998), pp. 153-170.

[10] T. Houghton-Larsen and K. Thomsen, Universal (co)homology theories , $K$ theory, 16 (1999), pp. 1-27.

[11] R.V. Kadison and J.R. Ringrose, Derivations and automorphisms of operator algebras, Comm. Math. Phys., 4 (1967), pp. 32-63.

[12] G. Kasparov, The operator $K$-functor and extensions of $C^{*}$-algebras, $I z v$. Akad. Nauk. SSSR, Ser. Mat., 44 (1980), pp. 571-636.

[13] G. Kasparov, Hilbert $C^{*}$-modules: theorems of Stinespring and Voiculescu, J. Oper. Th., 4 (1980), pp. 133-150.

[14] G. Kasparov, Equivariant KK-theory and the Novikov conjecture, Invent. Math., 91 (1988), pp. 513-572.

[15] E. Kirchberg, The classification of purely infinite $C^{*}$-algebras using Kasparov's theory, Preliminary version, Berlin 1994.

[16] K. Knudsen-Jensen and K. Thomsen, Elements of KK-theory, Birkhäuser, Boston, 1991.

[17] H. Lin, Stable Approximate Unitary Equivalence of Homomorphisms, $J$. Oper. Th., 47 (2003), pp. 343-368.

[18] H. Lin, Tracially AF $C^{*}$-algebras, Trans. Amer. Math. Soc., 353 (2001), pp. 693-722.

[19] V. Manuilov, K. Thomsen, Quasidiagonal extensions and sequentially trivial asymptotic homomorphisms, Adv. Math., 154 (2000), pp. 258-279.

[20] V. M. Manuilov and K. Thomsen, The Connes-Higson construction is an isomorphism, Preprint, 2000. 
[21] G. Nagy, On the K-groups of certain $C^{*}$-algebras used in E-theory, Proc. Amer. Math. Soc., 122(4) (1994), pp. 1125-1131.

[22] G.K. Pedersen, $C^{*}$-algebras and their Automorphism Groups, Academic Press, New York (1979).

[23] N.C. Phillips, A classification theorem for purely infinite simple $C^{*}$-algebras, Preprint, Oregon and Toronto, 1995.

[24] J. Rosenberg, The role of K-theory in non-commutative algebraic topology, Operator Algebras and K-theory. Contemporary Mathematics vol. 10 (Eds. R. Douglas and C. Schochet), Amer. Math. Soc., 1982.

[25] K. Thomsen, On absorbing extensions, Proc. Amer. Math. Soc., 129 (2001), pp. 1409-1417.

[26] K. Thomsen, Discrete asymptotic homomorphisms in E-theory and KKtheory, Math. Scand., 92 (2003), pp. 103-128.

[27] K. Thomsen, KK-equivalence and continuous bundles of $C^{*}$-algebras, New York J. Math., 6 (2000), pp. 285-306.

[28] K. Thomsen, On the KK-theory and E-theory of amalgamated free products of $C^{*}$-algebras, J. Func. Anal., 201 (2003), pp. 30-56.

[29] D. Voiculescu, A note on quasi-diagonal $C^{*}$-algebras and homotopy, Duke Math. J., 62 (1991), pp. 267-271.

Klaus Thomsen matkt@imf.au.dk

Institut for matematiske fag,

Ny Munkegade,

8000 Aarhus C,

Denmark

This article is available at http://intlpress.com/HHA/v8/n2/a2 